\title{
Research of Chaotic Dynamics of 3D Autonomous Quadratic Systems by Their Reduction to Special 2D Quadratic Systems
}

\author{
Vasiliy Belozyorov \\ Department of Applied Mathematics, Dnepropetrovsk National University, Gagarin's Avenue 72, Dnepropetrovsk 49050, Ukraine \\ Correspondence should be addressed to Vasiliy Belozyorov; belozvye@mail.ru
}

Received 3 December 2014; Revised 26 February 2015; Accepted 2 March 2015

Academic Editor: Jun-Juh Yan

Copyright (C) 2015 Vasiliy Belozyorov. This is an open access article distributed under the Creative Commons Attribution License, which permits unrestricted use, distribution, and reproduction in any medium, provided the original work is properly cited.

\begin{abstract}
New results about the existence of chaotic dynamics in the quadratic 3D systems are derived. These results are based on the method allowing studying dynamics of 3D system of autonomous quadratic differential equations with the help of reduction of this system to the special $2 \mathrm{D}$ quadratic system of differential equations.
\end{abstract}

\section{Introduction}

Chaos theory has successfully explained various phenomena from natural science to engineering and is being applied in many fields. In mathematics, chaos has become an important branch of dynamical systems. In physics, it has been used to illustrate complex behaviors, such as planetary orbits and fluid motion. In chemistry, it has been used to analyze the amounts of chemical compounds. In engineering, chaotic systems provide many potential applications, such as the secure communication, the chaos control, the information encryption, and the synchronization. In economics, it has been used to develop new types of econometric models and to analyze job selection. In psychology, chaos has been applied to cognitive and clinical psychology. In biology, it has been used to study insect populations.

There are two basic methods of search of chaotic systems. They are based either on establishment of the existence fact of a homoclinic orbit or on construction for the given system of a discrete map and proof of its state of chaos.

The construction of discrete maps for continuous dynamical systems is still small studied. Here basic results are contained, for example, in [1-11]. The main idea of these papers is that properties of the being created discrete maps describing the dynamics continuous dynamical systems are based on the well-known properties of the Ricker map $f(x)=$ $x \exp (r-x)$ and the logistic map $g(x)=r x(1-x)$. Our approach to research of chaos in 3D autonomous quadratic systems is also based on this idea.
There is a huge number of papers devoted to the search of homoclinic orbits in 3D systems of differential equations (see, e.g., [12-28]). Here, in our opinion, one of the most essential results was got in [17]. In this article the Fishing Principle allowing deciding a question about the existence of homoclinic orbits for large class of nonlinear systems was offered.

The most general approach at the study of chaos in the continuous 3D system consists in finding of a basin of attraction for this system. The simplest situation arises up then, when the basin of attraction is whole space $\mathbb{R}^{3}$. In other words, for existence of the basin it is sufficient that all solutions of the system were bounded at any initial data.

Notice that in the theory of dynamic systems, besides the methods mentioned above, methods connected with the invariant analysis of differential equations are also widespread (see, e.g., [29]). In [29] mentioned methods were applied for the study of dynamics of the Yang-Chen system. A construction of an invariant algebraic surface, which was introduced for research of the Lorenz system, is basis of these methods. Due to the concept invariant algebraic surface some new results on a behavior at infinity of the Yang-Chen system were got.

The present paper is a continuation of work [7]. It is needed to say that the basic idea of the represented work and article [7] consists in the reduction of 3D dynamic system to some 2D dynamic system. Howewer, in the present paper this idea was extended on other classes of quadratic 3D systems, 
which were not considered in [7] (see Section 4). In addition, the primary purpose of work [7] is a construction of implicit discrete mappings generating chaos in 3D dynamic systems. On the contrary, the obtaining of new existence conditions of chaos in 3D quadratic systems, which were not presented in work [7], is the main purpose of this paper.

At first we consider the following system:

$$
\begin{aligned}
\dot{x}(t)= & a_{11} x(t)+b_{11} y^{2}(t)+b_{12} y(t) z(t) \\
& +b_{22} z^{2}(t), \\
\dot{y}(t)= & \mu a_{21} x(t)+a_{22} y(t)+a_{23} z(t)+c_{11} x(t) y(t) \\
& +c_{12} x(t) z(t), \\
\dot{z}(t)= & \mu a_{31} x(t)-a_{23} y(t)+a_{22} z(t)+c_{21} x(t) y(t) \\
& +c_{11} x(t) z(t),
\end{aligned}
$$

where $a_{11}, a_{21}, \ldots, c_{11}, c_{21}$ are real numbers and $\mu \geq 0$ is a parameter. (If for some system in form (1) the summands $c_{11} x y$ and $c_{11} x z$ look like $d_{11} x y$ and $d_{22} x z$, then by suitable linear real replacement of variables $y \rightarrow p y_{1}+q z_{1}$ and $z \rightarrow-q y_{1}+p z_{1}$ it is always possible to obtain that, in the new variables $y_{1}, z_{1}$, we will have $d_{11}(p, q)=d_{22}(p, q)$.)

In paper [7] the theorem about boundedness of solutions of the general quadratic 3D systems of autonomous differential equations was proved. In application to system (1) this theorem looks as follows.

Theorem 1 (see [7, Theorem 3]). Suppose that for system (1) the following conditions are fulfilled:

(i) $a_{11}<0$;

(ii) the quadratic form $h(y, z)=b_{11} y^{2}+b_{12} y z+b_{22} z^{2} \neq \equiv 0$ is positive definite (negative definite);

(iii) the quadratic form $g(y, z)=c_{11} y^{2}+\left(c_{12}+c_{21}\right) y z+$ $c_{11} z^{2} \neq 0$ is negative definite (positive definite).

Then for any initial values and $\forall t \geq 0$ the solutions $x(t), y(t), z(t)$ of system (1) are bounded.

Without loss of generality, one will consider that the quadratic form $h(y, z)$ is positive definite and the quadratic form $g(y, z)$ is negative definite.

\section{On Existence of Limit Cycles in System (1)}

Let

$$
\dot{\mathbf{x}}(t)=\mathbf{G}(\mathbf{x}(t)), \quad \mathbf{x}(t) \in \mathbb{R}^{n}, \mathbf{G}(\mathbf{x}) \in \mathbb{R}^{n}, t \in \mathbb{R}
$$

be a system of ordinary autonomous differential equations and let $\mathbf{x}\left(t, \mathbf{x}_{0}\right)$ be a trajectory of this system with initial data $\mathbf{x}_{0} \in \mathbb{R}^{n}$. Here $\mathbf{G}(\mathbf{x}): \mathbb{R}^{n} \rightarrow \mathbb{R}^{n}$ is a continuous vectorfunction; $\mathbf{x}\left(0, \mathbf{x}_{0}\right)=\mathbf{x}_{0}$.

The trajectory $\mathbf{x}\left(t, \mathbf{x}_{0}\right)$ of system (2) is called periodic if there exists a constant $T>0$ such that

$$
\forall t \in \mathbb{R}, \quad \mathbf{x}\left(t+T, \mathbf{x}_{0}\right)=\mathbf{x}\left(t, \mathbf{x}_{0}\right) .
$$

Let $\mathbf{x}\left(t, \mathbf{y}_{0}\right)\left(\mathbf{x}_{0} \neq \mathbf{y}_{0}\right)$ be another trajectory of system (2) such that $\left\|\mathbf{x}_{0}-\mathbf{y}_{0}\right\|<\epsilon$, where the symbol $\|\mathbf{v}\|$ means the Euclidean norm of the vector $\mathbf{v} ; \epsilon \rightarrow 0$ is any positive small enough number.

The periodic trajectory $\mathbf{x}\left(t, \mathbf{x}_{0}\right)$ of system (2) is called isolated if for any positive small enough number $\epsilon>0$ there does not exist the periodic trajectory $\mathbf{x}\left(t, \mathbf{y}_{0}\right)$ such that $\left\|\mathbf{x}_{0}-\mathbf{y}_{0}\right\|<\epsilon$. The isolated periodic trajectory $\mathbf{x}\left(t, \mathbf{x}_{0}\right)$ of system (2) is called a limit cycle.

A set $\mathbb{M} \subset \mathbb{R}^{n}$ is said to be a positively invariant set with respect to (2) if from $\mathbf{x}_{0} \in \mathbb{M}$ it follows that $\mathbf{x}\left(t, \mathbf{x}_{0}\right) \in \mathbb{M} \forall t \geq$ 0.

A point $s \in \mathbb{R}^{n}$ is said to be a positive limit point of $\mathbf{x}\left(t, \mathbf{x}_{0}\right)$ if there is a sequence $\left\{t_{m}\right\}$, with $t_{m} \rightarrow \infty$ as $m \rightarrow \infty$, such that $\mathbf{x}\left(t_{m}, \mathbf{x}_{0}\right) \rightarrow \mathbf{s}$ as $m \rightarrow \infty$. The set $\mathbb{L}^{+}$of all positive limit points of $\mathbf{x}\left(t, \mathbf{x}_{0}\right)$ is called the positive limit set of $\mathbf{x}\left(t, \mathbf{x}_{0}\right)$.

Let $\mathbb{D} \subset \mathbb{R}^{n}$ be a compact set.

Lemma 2 (see [30, Lemma 3.1]). If solution $\mathbf{x}\left(t, \mathbf{x}_{0}\right)$ is bounded and belongs to $\mathbb{D}$, then its positive limit set $\mathbb{L}^{+}$is a nonempty, compact, invariant set. Moreover, $\mathbf{x}\left(t, \mathbf{x}_{0}\right) \rightarrow \mathbb{L}^{+}$as $t \rightarrow \infty$.

Let one for system (1) define new variables $\rho \geq 0$ and $\phi$ by the following formulas: $y=\rho \cos \phi$ and $z=\rho \sin \phi$. Then we obtain the new following system:

$$
\begin{aligned}
\dot{x}(t)= & a_{11} x \\
& +\left(b_{11} \cos ^{2} \phi+b_{12} \cos \phi \sin \phi+b_{22} \sin ^{2} \phi\right) \rho^{2}, \\
\dot{\rho}(t)= & \mu\left(a_{21} \cos \phi+a_{31} \sin \phi\right) x+a_{22} \rho \\
& +\left[c_{11}+\left(c_{12}+c_{21}\right) \cos \phi \sin \phi\right] x \rho, \\
\dot{\phi}(t)= & -a_{23}+\left[c_{21} \cos ^{2} \phi-c_{12} \sin ^{2} \phi\right] x \\
& +\mu\left(a_{31} \cos \phi-a_{21} \sin \phi\right)\left(\frac{x}{\rho}\right) .
\end{aligned}
$$

Further, one assumes in system (4) $c_{11}=c_{22}=p<0$, $a_{11}=a, a_{22}=b, a_{23}=c \neq 0$, and $a_{21}=a_{31}=0$. Then we get

$$
\begin{gathered}
\dot{x}(t)=a x(t)+\left(b_{11} \cos ^{2} \phi+b_{12} \cos \phi \sin \phi+b_{22} \sin ^{2} \phi\right) \rho^{2}, \\
\dot{\rho}(t)=b \rho+\left[p+\left(c_{12}+c_{21}\right) \cos \phi \sin \phi\right] x \rho \\
\dot{\phi}(t)=-c+\left[c_{21} \cos ^{2} \phi-c_{12} \sin ^{2} \phi\right] x .
\end{gathered}
$$

In order to determine the chaotic properties of system (1) system (5) will be used.

Theorem 3. Let system (5) be $b>0,\left(b_{11}-b_{22}\right)^{2}+b_{12}^{2} \neq 0$. Assume also that $\forall x \in \mathbb{R}$ the quadratic function $(b+p x)^{2}+$ $\left(c+c_{12} x\right)\left(c-c_{21} x\right) \neq 0$ and the conditions of Theorem 1 are valid. Then in system (5) there exists either a limit cycle or a limit torus. 
Proof. (A) Let us calculate Lyapunov's exponent $\Lambda$ for a real function $f(t)[7]$ :

$$
\Lambda[f]=\varlimsup_{t \rightarrow \infty} \frac{1}{t} \ln \left|\frac{f(t)}{f\left(t_{0}\right)}\right| .
$$

We take advantage of the following properties Lyapunov's exponents:

(a1) $\Lambda\left[f_{1}(t) \cdot f_{2}(t)\right] \leq \Lambda\left[f_{1}(t)\right]+\Lambda\left[f_{2}(t)\right]$;

(a2) if $m \geq 0$, then $\Lambda\left[t^{m}\right]=0$;

(a3) if $\Lambda[f(t)]<0$, then $\Lambda\left[\int_{t}^{\infty} f(\tau) d \tau\right] \leq \Lambda[f(t)]$;

(a4) $\Lambda\left[f_{1}(t)+f_{2}(t)\right] \leq \max \left(\Lambda\left[f_{1}(t)\right], \Lambda\left[f_{2}(t)\right]\right)$;

(a5) $\Lambda[d \cdot f(t)]=\Lambda[f(t)](d \neq 0)$.

Taking into account the boundedness of all solutions of system (5) we have $\Lambda[x] \leq 0, \Lambda[\rho] \leq 0$, and $\Lambda[\phi] \leq 0$.

Consider the function

$$
\begin{aligned}
h(\phi) & =b_{11} \cos ^{2} \phi+b_{12} \cos \phi \sin \phi+b_{22} \sin ^{2} \phi \\
& =\frac{b_{11}+b_{22}}{2}+\frac{b_{11}-b_{22}}{2} \cos 2 \phi+\frac{b_{12}}{2} \sin 2 \phi \\
& =\frac{b_{11}+b_{22}}{2}+\frac{\sqrt{\left(b_{11}-b_{22}\right)^{2}+b_{12}^{2}}}{2} \sin (2 \phi+\alpha),
\end{aligned}
$$

where

$$
\begin{aligned}
& \sin \alpha=\frac{b_{11}-b_{22}}{\sqrt{\left(b_{11}-b_{22}\right)^{2}+b_{12}^{2}}}, \\
& \cos \alpha=\frac{b_{12}}{\sqrt{\left(b_{11}-b_{22}\right)^{2}+b_{12}^{2}}} .
\end{aligned}
$$

It is clear that $h(\phi) \neq 0$, if

$$
\frac{\left|b_{11}+b_{22}\right|}{\sqrt{\left(b_{11}-b_{22}\right)^{2}+b_{12}^{2}}}>1 \text {. }
$$

In this case for the function $h(\phi(t))$ Lyapunov's exponent $\Lambda(h)=0$. If

$$
\frac{\left|b_{11}+b_{22}\right|}{\sqrt{\left(b_{11}-b_{22}\right)^{2}+b_{12}^{2}}} \leq 1,
$$

then there exists the point $\phi=\gamma$ such that $h(\gamma)=0$. In this case $\Lambda(h)=-\infty$.

We write the first equation of system (5) in the following integral form:

$$
x(t)=x_{0} \exp (a t)+\int_{0}^{t} \exp (a(t-\tau)) \rho^{2}(\tau) h(\tau) d \tau .
$$

From here and (al) it follows that $\Lambda\left(\exp (a t) \rho^{2} h\right) \leq$ $\Lambda(\exp (a t))+\Lambda\left(\rho^{2}\right)+\Lambda(h) \leq a+0+0=a$ or $\Lambda\left(\exp (a t) \rho^{2} h\right)=$ $-\infty$. Thus, from (a2) and (a3) it follows that

$$
\Lambda\left[\int_{t}^{\infty} \exp (a(t-\tau)) \rho^{2}(\tau) h(\tau) d \tau\right] \leq a .
$$

Therefore, from boundedness of $x(t)$ and (a4), we have $\Lambda[x(t)] \leq a$.

From the third equation of system (5), we get

$$
\phi(t)=\phi_{0}-c t+\int_{0}^{t}\left[c_{21} \cos ^{2} \phi(\tau)-c_{12} \sin ^{2} \phi(\tau)\right] x(\tau) d \tau .
$$

Then from (a3)-(a5) it follows that

$$
\begin{aligned}
\Lambda[\phi(t)] \leq & \Lambda\left[\phi_{0}-c t\right] \\
& +\Lambda\left[\int_{0}^{\infty}\left[c_{21} \cos ^{2} \phi(\tau)-c_{12} \sin ^{2} \phi(\tau)\right] x(t) d t\right] \\
\leq & \max (0, a)=0 .
\end{aligned}
$$

From the condition $(b+p x)^{2}+\left(c+c_{12} x\right)\left(c-c_{21} x\right) \neq 0$ it follows that the origin is a unique equilibrium of system (5). As $b>0$, then the origin is a saddle-focus, and we have $\Lambda[x] \leq a<0, \Lambda[\rho] \leq 0$, and $\Lambda[\phi]=0$.

(B) According to the conditions of Theorem 3 solutions $x(t)$ and $\rho(t)$ of system (5) are bounded at any initial data. Consequently for the concrete solution $\mathbf{x}\left(t, \mathbf{x}_{0}\right)$ (in polar coordinates it is $(x(t), \rho(t), \phi(t)))$ there is a nonempty compact invariant set $\mathbb{L}^{+}$(see Lemma 2$)$.

Assume that the vector solution $\left(x^{+}(t), \rho^{+}(t), \phi^{+}(t)\right) \in \mathbb{L}^{+}$. By virtue of boundedness of any solutions $x(t)$ and $\rho(t)$ of system (5) there must be points $t_{k}$ such that $\dot{x}^{+}\left(t_{k}\right)=0$, $k=1,2, \ldots$. Therefore, from the first equation of system (5) and condition $\dot{x}^{+}\left(t_{k}\right)=0$, we have $h\left(\phi^{+}\left(t_{k}\right)\right)=$ $-a x^{+}\left(t_{k}\right) / \rho^{+2}\left(t_{k}\right)>0$.

We will consider that $x_{0}^{+}>0$. Then from this condition and the first equation of system (5) it follows that $\forall t>$ $0 x(t)>0$ (see the proof of Theorem 3 [7]). From the condition $\left(b_{11}-b_{22}\right)^{2}+b_{12}^{2} \neq 0$ and $\pi$-periodicity of the nonnegative function $h(\phi)$ it follows that the relation $x^{+}\left(t_{k}\right) / \rho^{+2}\left(t_{k}\right)$ is also $\pi$-periodic, $k=1,2, \ldots$.

There are only two possibilities:

(b1) the functions $x^{+}(t)$ and $\rho^{+}(t)$ are periodic;

(b2) the functions $x^{+}(t)$ and $\rho^{+}(t)$ are not periodic.

Assume that condition (b2) takes place. Then there does not exist a number $T>0$ such that $\forall t \geq 0 \quad \rho^{+}(t+T)=\rho^{+}(t)$. Consequently the inequality

$$
b+\left[p+\left(c_{12}+c_{21}\right) \cos \phi^{+}(t) \sin \phi^{+}(t)\right] x^{+}(t) \neq 0
$$

follows from the second equation of system (5). It means that the function $\rho^{+}(t)$ is unbounded. We derived the contradiction. Consequently the condition (b1) must be valid.

In (A) for any solutions $(x(t), \rho(t), \phi(t))$ the conditions $\Lambda[x] \leq a<0, \Lambda[\rho] \leq 0$, and $\Lambda[\phi]=0$ were got. In addition, we know that the periodic solution $\left(x^{+}(t), \rho^{+}(t), \phi^{+}(t)\right)$ exists. From here it follows that in system (5) there is either a limit cycle $\mathbb{L}^{+}=\left(x^{+}(t), \rho^{+}(t), \phi^{+}(t)\right)$ or a limit torus (if $\Lambda[\rho]=0$ ). This completes the proof of Theorem 3 . 
Theorem 4. Let system (1) be $\mu \neq 0$. Assume that all conditions of Theorem 3 are valid. If the parameter $\mu>0$ is small enough, then in system (1) there exists a limit cycle.

Proof. (C) According to Theorem 3 at $\mu=0$ in system (1) there is a limit cycle. We designate this cycle as $C^{+}=$ $\left(x^{+}(t), y^{+}(t), z^{+}(t)\right)^{T} \subset \mathbb{R}^{3}$. Let $T=T(0)$ be a period of the cycle $C^{+}$.

Compute Lyapunov's exponents $\lambda_{1}(\mu), \lambda_{2}(\mu)$, and $\lambda_{3}(\mu)$ on cycle $C^{+}$. From Theorems 1 and 3 it follows that

(c1) at $\mu \geq 0$ and any initial values all solutions of system (1) are bounded;

(c2) system (1) at $\mu=0$ has a unique equilibrium point; it is a saddle-focus (an unstable point);

(c3) system (1) at $\mu=0$ has a unique stable limit cycle $\mathrm{C}^{+}$; all solutions of system (1) at any initial values are attracted to this cycle.

Thus, from item (c1) it follows that Lyapunov's exponents of cycle $C^{+}$are as follows: $\lambda_{1}(0)=0, \lambda_{2}(0)<0$, and $\lambda_{3}(0)<0$.

Now let $\mu$ be the sufficiently small positive parameter. Then, by virtue of continuity of Lyapunov's exponents for the small enough $\mu$, it is possible to consider that $\lambda_{2}(\mu)<$ $0, \lambda_{3}(\mu)<0$. Consequently for the exponent $\lambda_{1}(\mu)$ such following suppositions take place.

$\lambda_{1}(\mu)>0$; it contradicts with item (c1) (indeed, the presence of positive Lyapunov's exponent will result in unlimited growth of solutions of system (1)).

$\lambda_{1}(\mu)<0$; in this case all solutions must converge to the origin (it must be a stable node); we get the contradiction with item (c2).

Thus, there is only the case $\lambda_{1}(\mu)=0$; it means that for the small enough $\mu>0$ in system (1) there is a stable limit cycle different from $C^{+}$. The period of this cycle is equal to $T(\mu)=m T(0)$, where $m>1$ is integer.

\section{Chaotic Behavior of System (4)}

In this section we will consider that all conditions of Theorems 3 and 4 are valid.

Let system (4) be $c_{11}=c_{22}=p<0,\left(c_{12}+c_{21}\right) \cos \phi \sin \phi=$ $d(\phi), a_{11}=a<0, a_{22}=b>0$, and $a_{23}=c>0$. Then we derive the following system:

$$
\begin{gathered}
\dot{x}(t)=a x+\left(b_{11} \cos ^{2} \phi+b_{12} \cos \phi \sin \phi+b_{22} \sin ^{2} \phi\right) \rho^{2} \\
\dot{\rho}(t)=\mu\left(a_{21} \cos \phi+a_{31} \sin \phi\right) x+b \rho+(p+d(\phi)) x \rho \\
\dot{\phi}(t)=-c+\left[c_{21} \cos ^{2} \phi-c_{12} \sin ^{2} \phi\right] x \\
+\mu\left(a_{31} \cos \phi-a_{21} \sin \phi\right)\left(\frac{x}{\rho}\right),
\end{gathered}
$$

where initial values $x_{0}>0, \rho_{0}>0$, and $\phi_{0}$ are given.

Theorem 5. Let $\mu^{*}$ be the maximal positive parameter such that for system (16) and any $\mu \in\left[0, \mu^{*}\right)$ all conditions of
Theorems 3 and 4 are valid. Assume that there exists the parameter $\mu_{c}^{*} \in\left(0, \mu^{*}\right)$ such that the condition

$$
\liminf _{t \rightarrow \infty} \rho(t)=0
$$

takes place. Then in system (16) there is chaotic dynamics.

Proof. (D) Let the condition (17) be valid. Investigate a behavior of the function $\rho(t)$ at the increase of $\mu$.

(d1) At first we show that if $\rho_{0}>0$, then $\rho(t) \geq 0$. Assume that there exists $t^{*}>0$ such that $\lim _{t \rightarrow t^{*}} \rho\left(t^{*}\right) \rightarrow 0$.

Compute the second derivative of the function $\rho(t)$ in the point $t^{*}$. We get

$$
\begin{aligned}
\lim _{t \rightarrow t^{*}} \ddot{\rho}(t)= & \mu^{2}\left(a_{21} \sin \phi\left(t^{*}\right)-a_{31} \cos \phi\left(t^{*}\right)\right)^{2} \frac{x^{2}\left(t^{*}\right)}{\rho\left(t^{*}\right)} \\
& +\Phi\left(t^{*}\right),
\end{aligned}
$$

where $\Phi(t)$ is a continuous function such that $\lim _{t \rightarrow t^{*}} \Phi(t)$ is a finite number. Thus, if $\rho_{0}>0$ and $\rho(t) \rightarrow 0$, then $\lim _{t \rightarrow t^{*}} \ddot{\rho}(t) \rightarrow \infty$ and the function $\rho(t)$ in the point $t^{*}$ is concave. Therefore, $t^{*}$ is a minimum point, $\lim _{t \rightarrow t^{*}} \dot{\rho}(t)=0$, and $\forall t>0 \rho(t) \geq 0$. In addition, from second equation of system (16) we have $a_{21} \cos \phi\left(t^{*}\right)+a_{31} \sin \phi\left(t^{*}\right)=0$.

(d2) The solution of the second equation of system (16) can be represented in the following integral form:

$$
\begin{array}{r}
\rho(t) \\
=\exp \left[\int_{0}^{t}(b+(p+d(\phi(\omega))) x(\omega)) d \omega\right] \\
\times\left[\rho_{0}+\mu \int_{0}^{t}\left(a_{21} \cos \phi(\tau)+a_{31} \sin \phi(\tau)\right) x(\tau)\right. \\
\cdot \exp \left[-\int_{0}^{\tau}(b+(p+d(\phi(\tau)))\right. \\
\cdot x(\tau)) d \tau] d \tau], \\
t>\tau,
\end{array}
$$

where $\forall \phi(\tau)(p+d(\phi(\tau)))<0$.

Suppose that in formula (19) the variable $t$ takes two values: $t_{k}$ and $t_{k+1}, t_{k}<t_{k+1}$. Then we can define the numbers $\rho\left(t_{k}\right)=\rho_{k}>0, \rho\left(t_{k+1}\right)=\rho_{k+1}>0, x\left(t_{k}\right)=x_{k}>0$, and $x\left(t_{k+1}\right)=x_{k}>0$. Introduce the following designation:

$$
\Delta(t)=\exp \left[\int_{t_{0}}^{t}(b+(p+d(\phi(\omega))) x(\omega)) d \omega\right] .
$$

Then from formula (19) it follows that

$$
\begin{aligned}
\rho_{k+1}= & \rho_{0} \Delta\left(t_{k+1}\right) \\
& +\mu \Delta\left(t_{k+1}\right) \int_{t_{0}}^{t_{k+1}} \Delta(-\tau) x(\tau)\left(a_{21} \cos \phi(\tau)\right. \\
& \left.+a_{31} \sin \phi(\tau)\right) d \tau,
\end{aligned}
$$




$$
\begin{aligned}
\rho_{k}= & \rho_{0} \Delta\left(t_{k}\right) \\
& +\mu \Delta\left(t_{k}\right) \int_{t_{0}}^{t_{k}} \Delta(-\tau) x(\tau)\left(a_{21} \cos \phi(\tau)\right. \\
& \left.+a_{31} \sin \phi(\tau)\right) d \tau
\end{aligned}
$$

The first equation of system (16) on interval $\left[t_{k}, t_{k+1}\right]$ may be also written in the integral form as

$$
\begin{aligned}
& x_{k+1} \\
& =x_{k} \exp \left(a\left(t_{k+1}-t_{k}\right)\right) \\
& +\int_{t_{k}}^{t_{k+1}} \exp \left(a\left(t_{k+1}-\tau\right)\right) h(\cos \phi(\tau), \sin \phi(\tau)) \rho^{2}(\tau) d \tau, \\
& t_{k}<\tau<t_{k+1} .
\end{aligned}
$$

(d3) Let $x\left(t_{i}\right)=x_{i}, \rho\left(t_{i}\right)=\rho_{i}$, and $\phi\left(t_{i}\right)=\phi_{i}$, where $t_{i}$ are roots of the first equation $\dot{x}\left(t_{i}\right)=a x+\left(b_{11} \cos ^{2} \phi+\right.$ $\left.b_{12} \cos \phi \sin \phi+b_{22} \sin ^{2} \phi\right) \rho^{2}=0$ of system (16), $i=1,2, \ldots$ [7]. We can consider that $\cdots<-\phi_{i-1}<-\phi_{i}<-\phi_{i+1}<-\phi_{i+2}<\cdots$ and $t_{0}<t_{1}<\cdots<t_{i}<t_{i+1}<\cdots$.

We can also assume that the variants taking place, $t_{k}, t_{k+1}$, $t_{k+2}, \ldots$, are sequential maximum (or a point of inflection), minimum (or a point of inflection), and maximum (or a point of inflection) of the function $\rho(t)$. As the function $\left(a_{21} \cos \phi+\right.$ $\left.a_{31} \sin \phi\right)$ is $2 \pi$-periodic, then $\phi\left(t_{k+1}\right)-\phi\left(t_{k}\right)=-\pi$.

According to Theorems 3 and 4 for sufficiently small $\mu \geq 0$ solutions $x(t)$ and $\rho(t)$ of system (16) are bounded. Therefore, we can consider that the integer $k$ is large enough in order that the trajectory of system (16) got on an attractor (it is a limit cycle). Thus, we have

$$
\dot{x}\left(t_{k}\right)=0 \longrightarrow x\left(t_{k}\right)=-\frac{1}{a} h\left(\cos \phi\left(t_{k}\right), \sin \phi\left(t_{k}\right)\right) \rho^{2}\left(t_{k}\right) .
$$

Taking into account formula (24) and the known first Theorem about Mean Value we can rewrite formula (22) as

$$
\begin{aligned}
\rho_{k+1} \approx & \Delta\left(t_{k+1}\right) \Delta\left(-t_{k}\right) \rho_{k} \\
& +\mu \frac{\Delta\left(t_{k+1}\right) \Delta\left(-\xi_{k}\right) h\left(\cos \phi\left(\xi_{k}\right), \sin \phi\left(\xi_{k}\right)\right) \rho^{2}\left(\xi_{k}\right)}{-a} \\
& \cdot \int_{t_{k}}^{t_{k+1}}\left(a_{21} \cos \phi(\tau)+a_{31} \sin \phi(\tau)\right) d \tau,
\end{aligned}
$$

where $t_{k}<\xi_{k}<t_{k+1}$ and $\forall \tau \in\left(t_{k}, t_{k+1}\right)$, we have $a_{21} \cos \phi(\tau)+$ $a_{31} \sin \phi(\tau) \neq 0$. (d4) It is clear that in (25) we have $\mu>0, \Delta\left(t_{k+1}\right) \Delta\left(-\xi_{k}\right)>$ 0 , and $h\left(\cos \phi\left(\xi_{k}\right), \sin \phi\left(\xi_{k}\right)\right)>0$. In this case we can introduce the following designations:

$$
\begin{gathered}
\Delta\left(t_{k+1}\right) \Delta\left(-t_{k}\right)=\theta(\mu)>0, \\
\frac{h\left(\cos \phi\left(t_{k}\right), \sin \phi\left(t_{k}\right)\right) \rho^{2}\left(t_{k}\right)}{-a}=\psi(\mu)>0, \\
\int_{t_{k}}^{t_{k+1}}\left(a_{21} \cos \phi(\tau)+a_{31} \sin \phi(\tau)\right) d \tau=-\omega(\mu)<0,
\end{gathered}
$$

where the functions $\theta(\mu), \psi(\mu)$, and $\omega(\mu)$ do not depend on $k$. The last inequality is stipulated to those in formula (25). We obtain $\lim _{t \rightarrow t_{m}^{*}} \rho\left(t_{m}^{*}\right) \rightarrow 0$ at some $t_{m}^{*}$, if and only if the second summand of this formula is negative. It is obvious that at the implementation of condition (17) this restriction will be satisfied. In addition, as shown in [31] (Lemma 10) the condition $-\omega(\mu)<0$ is necessary for existence in the system

$$
\begin{aligned}
& \dot{x}(t)=a x+\left(b_{11} \cos ^{2} \phi+b_{12} \cos \phi \sin \phi+b_{22} \sin ^{2} \phi\right) \rho^{2}, \\
& \dot{\rho}(t)=\mu\left(a_{21} \cos \phi+a_{31} \sin \phi\right) x+b \rho+(p+d(\phi)) x \rho
\end{aligned}
$$

of a separatrix loop. This peculiarity of system (27) will be used in future for research of generalizations of system (16).

Further, process (25) may be represented in the following form:

$$
\rho_{k+1}=\theta(\mu) \cdot\left(\rho_{k}-\mu \psi(\mu) \omega(\mu) \rho_{k}^{2}\right), \quad k=0,1, \ldots
$$

Introduce the new variable $v_{k}=\mu \psi(\mu) \omega(\mu) \rho_{k}$, where $\mu \psi(\mu) \omega(\mu)>0$. Then process (28) will be generated by known logistic map as follows:

$$
v_{k+1}=\Psi\left(v_{k}, \delta\right) \equiv \delta \cdot v_{k} \cdot\left(1-v_{k}\right), \quad k=0,1, \ldots,
$$

where $\delta=\theta(\mu)>0$.

Let $v_{k}^{*}$ be the minimal fixed point of mapping $\Psi^{(k)}(v, \delta)$. It is known that if $\delta=\delta^{*} \in(3.84 \div 4)$, then the logistic map $\Psi(v, \delta)$ is chaotic and $\lim _{k \rightarrow \infty} v_{k}^{*}\left(\delta^{*}\right)=0$. Hence, from condition (17) it follows that there exists the parameter $\mu_{c}^{*} \in\left(0, \mu^{*}\right)$ such that at $\mu=\mu_{c}^{*}$ process (28) generates the subsequence $\rho_{m_{1}}, \ldots, \rho_{m_{k}}, \ldots$, for which $\rho_{m_{k}}=$ $\lim _{t \rightarrow t_{m_{k}}^{*}} \rho\left(t_{m_{k}}^{*}\right)<\epsilon \approx 0, k \geq 1$. It means that in system (16) there is chaotic dynamics.

\section{Generalizations}

Consider 3D autonomous system

$$
\dot{\mathbf{x}}(t)=H \mathbf{x}+\mathbf{f}(\mathbf{x})
$$

where $\mathbf{x}=(x, y, z)^{T}$ and $H=\left\{h_{i j}\right\}, i, j=1, \ldots, 3$, is a real $(3 \times 3)$-matrix. Consider

$$
\begin{gathered}
\mathbf{f}(\mathbf{x})=\left(f_{1}(x, y, z), f_{2}(x, y, z), f_{3}(x, y, z)\right)^{T} \in \mathbb{R}^{3}, \\
f_{1}(x, y, z)=a_{12} x y+a_{22} y^{2}+a_{13} x z+a_{23} y z+a_{33} z^{2}, \\
f_{2}(x, y, z)=b_{12} x y+b_{22} y^{2}+b_{13} x z+b_{23} y z+b_{33} z^{2}, \\
f_{3}(x, y, z)=c_{12} x y+c_{22} y^{2}+c_{13} x z+c_{23} y z+c_{33} z^{2}
\end{gathered}
$$

are real quadratic polynomials. 
Suppose that the matrix

$$
\left(\begin{array}{ll}
a_{12} & a_{13} \\
b_{12} & b_{13} \\
c_{12} & c_{13}
\end{array}\right)
$$

has rank 1 or 2 . Then by suitable linear transformations of variables $x \rightarrow x_{1}+\alpha_{1} y_{1}+\alpha_{2} z_{1}\left(\alpha_{1}, \alpha_{2} \in \mathbb{R}\right), y \rightarrow y_{1}$, and $z \rightarrow z_{1}$ system (30) can be represented in the same form (30), where $H \rightarrow \bar{H}=\left\{\overline{h_{i j}}\right\}, i, j=1, \ldots, 3$, and

$$
\begin{gathered}
f_{1}\left(x_{1}, y_{1}, z_{1}\right)=\bar{a}_{22} y_{1}^{2}+\bar{a}_{23} y_{1} z_{1}+\bar{a}_{33} z_{1}^{2}, \\
f_{2}\left(x_{1}, y_{1}, z_{1}\right) \\
=\bar{b}_{12} x_{1} y_{1}+\bar{b}_{22} y_{1}^{2}+\bar{b}_{13} x_{1} z_{1}+\bar{b}_{23} y_{1} z_{1}+\bar{b}_{33} z_{1}^{2}, \\
f_{3}\left(x_{1}, y_{1}, z_{1}\right) \\
=\bar{c}_{12} x_{1} y_{1}+\bar{c}_{22} y_{1}^{2}+\bar{c}_{13} x_{1} z_{1}+\bar{c}_{23} y_{1} z_{1}+\bar{c}_{33} z_{1}^{2},
\end{gathered}
$$

and $\bar{b}_{12} \neq 0$ or $\bar{b}_{13} \neq 0$.

Thus, without loss of generality, we will study system (30) under the following conditions:

$$
a_{12}=a_{13}=0, \quad b_{12} \vee b_{13} \neq 0 .
$$

Introduce into system (30) (taking into account (34)) new variables $\rho$ and $\phi$ under the formulas: $y=\rho \cos \phi, z=$ $\rho \sin \phi$, where $\rho>0$. Then, after replacement of variables and multiplication of the second and third equations of system (30) on the matrix

$$
\left(\begin{array}{cc}
\cos \phi(t) & \sin \phi(t) \\
-\frac{(\sin \phi(t))}{\rho(t)} & \frac{(\cos \phi(t))}{\rho(t)}
\end{array}\right)
$$

we get

$$
\begin{aligned}
\dot{x}(t)= & h_{11} x(t)+\left(h_{12} \cos \phi(t)+h_{13} \sin \phi(t)\right) \rho(t) \\
+ & \left(a_{22} \cos ^{2} \phi(t)+a_{23} \cos \phi(t) \sin \phi(t)\right. \\
& \left.\quad+a_{33} \sin ^{2} \phi(t)\right) \rho^{2}(t) \\
\dot{\rho}(t)= & \left(h_{21} \cos \phi(t)+h_{31} \sin \phi(t)\right) x(t) \\
+ & {\left[h_{22} \cos ^{2} \phi(t)+h_{33} \sin ^{2} \phi(t)\right.} \\
& \left.+\left(h_{32}+h_{23}\right) \cos \phi(t) \sin \phi(t)\right] \rho(t) \\
+ & {\left[b_{12} \cos ^{2} \phi(t)+\left(b_{13}+c_{12}\right) \cos \phi(t) \sin \phi(t)\right.} \\
& \left.\quad+c_{13} \sin ^{2} \phi(t)\right] x(t) \rho(t) \\
+ & {\left[b_{22} \cos ^{3} \phi(t)+\left(b_{23}+c_{22}\right) \cos ^{2} \phi(t) \sin ^{2}(t)\right.} \\
& \left.+\left(b_{33}+c_{23}\right) \cos \phi(t) \sin ^{2} \phi(t)+c_{33} \sin ^{3} \phi(t)\right] \\
+ & \rho^{2}(t),
\end{aligned}
$$

$$
\begin{aligned}
\dot{\phi}(t)= & \left.-h_{21} \sin \phi(t)+h_{31} \cos \phi(t)\right) \frac{x(t)}{\rho(t)} \\
+ & {\left[h_{32} \cos ^{2} \phi(t)-h_{23} \sin ^{2} \phi(t)\right.} \\
& \left.+\left(h_{33}-h_{22}\right) \cos \phi(t) \sin \phi(t)\right] \\
+ & {\left[b_{13} \sin ^{2} \phi(t)+\left(b_{12}-c_{13}\right) \sin \phi(t) \cos \phi(t)\right.} \\
& \left.\quad-c_{12} \cos ^{2} \phi(t)\right] x(t) \\
- & {\left[-c_{22} \cos ^{3} \phi(t)+\left(b_{22}-c_{23}\right) \cos ^{2} \phi(t) \sin \phi(t)\right.} \\
& \left.+\left(b_{23}-c_{33}\right) \cos \phi(t) \sin ^{2} \phi(t)+b_{33} \sin ^{3} \phi(t)\right] \rho(t) .
\end{aligned}
$$

Consider the first and second equations of system (36). One has

$$
\begin{aligned}
\dot{x}(t)= & h_{11} x+f(\cos \phi, \sin \phi) \rho+f_{22}(\cos \phi, \sin \phi) \rho^{2}, \\
\dot{\rho}(t)= & g(\cos \phi, \sin \phi) x+h(\cos \phi, \sin \phi) \rho \\
& +g_{12}(\cos \phi, \sin \phi) x \rho+g_{22}(\cos \phi, \sin \phi) \rho^{2},
\end{aligned}
$$

where $\phi$ is a real parameter. Consider

$$
\begin{gathered}
f(\cos \phi, \sin \phi)=h_{12} \cos \phi+h_{13} \sin \phi, \\
f_{22}(\cos \phi, \sin \phi) \\
=a_{22} \cos ^{2} \phi+a_{23} \cos \phi \sin \phi+a_{33} \sin ^{2} \phi, \\
g(\cos \phi, \sin \phi)=h_{21} \cos \phi+h_{31} \sin \phi, \\
h(\cos \phi, \sin \phi) \\
=h_{22} \cos ^{2} \phi+h_{33} \sin ^{2} \phi+\left(h_{23}+h_{32}\right) \cos \phi \sin \phi, \\
g_{12}(\cos \phi, \sin \phi) \\
=b_{12} \cos ^{2} \phi+\left(b_{13}+c_{12}\right) \cos \phi \sin \phi+c_{13} \sin ^{2} \phi, \\
g_{22}\left(\cos ^{2}, \sin ^{2}\right) \\
=b_{22} \cos ^{3} \phi+\left(b_{23}+c_{22}\right) \cos ^{2} \phi \sin ^{2} \\
+\left(b_{33}+c_{23}\right) \cos \phi \sin ^{2} \phi+c_{33} \sin ^{3} \phi
\end{gathered}
$$

(System (27) is a special case of system (37).)

Let $\Delta_{1}(\cos \phi, \sin \phi) \equiv h_{11} \cdot h(\cos \phi, \sin \phi)-$ $f(\cos \phi, \sin \phi) \cdot g(\cos \phi, \sin \phi), \quad \Delta_{2}(\cos \phi, \sin \phi) \quad \equiv$ $f_{22}(\cos \phi, \sin \phi) \cdot g_{12}(\cos \phi, \sin \phi)$, and $\Delta_{3}(\cos \phi, \sin \phi) \equiv$ $g_{22}^{2}(\cos \phi, \sin \phi)+4 \Delta_{2}(\cos \phi, \sin \phi)$ be the bounded functions. In addition, we will consider that $\Delta_{1}(u, v)$ and $\Delta_{2}(u, v)$ are nonsingular forms of the variables $u \equiv \cos \phi$ and $v \equiv \sin \phi$.

Theorem 6. Let $h_{11}<0$. Suppose also that $\forall \phi \in \mathbb{R}$ for system (37) with the following conditions:

(i) either $\Delta_{1}(\cos \phi, \sin \phi) \leq 0$ or $\Delta_{1}(\cos \phi$, $\sin \phi)$ is periodic alternating in sign on $(-\infty, \infty)$ function; 
(ii) either $\Delta_{2}(\cos \phi, \sin \phi) \leq 0$ and $\Delta_{3}(\cos \phi, \sin \phi) \leq 0$ or $\Delta_{2}(\cos \phi$, sin $\phi)$ and $\Delta_{3}(\cos \phi, \sin \phi)$ are periodic nonpositive functions are fulfilled.

Assume that the condition

$$
\liminf _{t \rightarrow \infty} \rho(t)=0
$$

is also valid. (From this condition it follows that $\forall \epsilon>0 \exists t_{m}^{*}$ such that $\rho\left(t_{m}^{*}\right)<\epsilon$.)

Then in system (36) (and system (30)) there is chaotic dynamics.

Proof. (E) Above it was shown that for system (30) can be always found the set of parameters such that at some values of these parameters $\mathbb{S}=\left\{\bar{h}_{11}, \ldots, \bar{c}_{33}\right\}$ in system (30) there is a limit cycle (see Theorem 4). Suppose that the parameters of system (30) have values from the set $\mathbb{S}$. Then from third equation of system (36) it follows that the right part of this equation is a periodic function (let it be $\omega(t)$ ).

We take advantage of the next known result. Let $T$ be a period of the continuous periodic function $\omega(t)$. Then from third equation of system (36), we get

$$
\phi(t)=\int_{t_{0}}^{t} \omega(\tau) d \tau=\Lambda(t)+\Lambda_{0}(t)
$$

where $\Lambda(t)$ is $T$-periodic function, $\Lambda_{0}(t)=k\left(t-t_{0}\right)+d$ is a linear function, and $k$ and $d$ are constants.

Therefore, in system (36) we can consider that $\phi(t) \sim k t$ (in other words $\lim _{t \rightarrow \infty} \phi(t) / t=k$ ). Thus, in system (37) it is possible to do the replacement $\phi(t) \rightarrow k t$. The coefficients of system (37) are periodic functions and therefore these coefficients are bounded. Thus, $\forall \phi \in \mathbb{R}$ the conditions of Theorem 6 coincide with the conditions of Theorem 1 [31]. From here it follows that for any initial values solutions of system (37) are uniformly bounded.

It is clear that without loss of generality we can consider that $g_{22}(\cos \phi, \sin \phi) \equiv 0$. Then we must consider two cases: (e1) $g(\cos \phi, \sin \phi) \neq \equiv$ and $(\mathrm{e} 2) g(\cos \phi$, $\sin \phi) \equiv 0$.

(e1) In this case the proof of Theorem 6 almost fully repeats the proof of Theorem 5. This process amounts to construction of the logistic map and proof of its state of chaos.

(e2) In this case the proof of Theorem 6 (without loss of generality!) is very convenient to show on a concrete example.

Consider the well-known Lorenz system

$$
\begin{gathered}
\dot{x}(t)=-\sigma x(t)+\sigma y(t), \\
\dot{y}(t)=r x(t)-y(t)-x(t) z(t), \\
\dot{z}(t)=-b z(t)+x(t) y(t),
\end{gathered}
$$

where parameters $\sigma=10, b=8 / 3$, and $r=24.74$. (It is the classic values of parameters at which system (41) demonstrates a chaotic behavior.)
We pass in system (41) to the coordinates $(z, \rho, \phi)$ under the formulas: $z=z, x=\rho \cos \phi$, and $y=\rho \sin \phi$, where $\rho>0$. Then we obtain

$$
\begin{gathered}
\dot{z}(t)=-b z(t)+\frac{\rho^{2}(t)}{2} \sin 2 \phi(t), \\
\dot{\rho}(t)=\left(-\frac{\sigma+1}{2}-\frac{\sigma-1}{2} \cos 2 \phi(t)+\frac{\sigma+r}{2} \sin 2 \phi(t)\right) \rho(t) \\
-\frac{z(t) \rho(t)}{2} \sin 2 \phi(t), \\
\dot{\phi}(t)=\frac{\sigma-1}{2} \sin 2 \phi(t)+\frac{\sigma+r}{2} \cos 2 \phi(t) \\
+\frac{r-\sigma}{2}-\frac{z(t)}{2}(1+\cos 2 \phi(t)) .
\end{gathered}
$$

By definition, we have $\rho(t) \geq 0$. Let $z(t)=z_{1}(t)+(\sigma+r)$ and $\rho(t)=\rho_{1}(t)$ be new variables. Then from the first and second equations of system (42) it follows that

$$
\begin{aligned}
\dot{z}_{1}(t) & =-b\left(z_{1}(t)+(\sigma+r)\right)+\frac{\rho_{1}^{2}(t)}{2} \sin 2 \phi(t) \\
& \leq-b z_{1}(t)+\frac{\rho_{1}^{2}(t)}{2} \sin 2 \phi(t), \\
\dot{\rho}_{1}(t) & \leq\left(\frac{\sigma+r}{2} \sin 2 \phi(t)\right) \rho_{1}(t)-\frac{z_{1}(t) \rho_{1}(t)}{2} \sin 2 \phi(t) \\
& =-\frac{z_{1}(t) \rho_{1}(t)}{2} \sin 2 \phi(t) .
\end{aligned}
$$

Consider the system

$$
\begin{gathered}
\dot{z}_{1}(t)=-b z_{1}(t)+\frac{\rho_{1}^{2}(t)}{2} \sin 2 \phi(t), \\
\dot{\rho}_{1}(t)=-\frac{z_{1}(t) \rho_{1}(t)}{2} \sin 2 \phi(t) .
\end{gathered}
$$

It is clear that the function $V(t)=z_{1}^{2}(t)+\rho_{1}^{2}(t)$ is Lyapunov's function for system (44). (Really, $V(t) \geq 0$ and $\dot{V}(t)=2 \dot{z}_{1}(t) z_{1}(t)+2 \dot{\rho}_{1}(t) \rho_{1}(t)=-2 b z_{1}^{2}(t) \leq 0$.) Thus, at $t \geq 0$ the solutions $z_{1}(t)$ and $\rho_{1}(t)$ of system (44) are bounded. Since $z(t)=z_{1}(t)+(\sigma+r)$ and $\rho(t)=\rho_{1}(t)$, then with the help of Comparison Principle [30] it is easy to check that solutions $z(t)$ and $\rho(t)$ of system (42) are also bounded at $t \geq 0$.

As well as in Section 3, we can assume that $t_{k}, t_{k+1}$, $t_{k+2}, \ldots$ are sequential maximum (or a point of inflection), minimum (or a point of inflection), and maximum (or a point of inflection) of the function $z(t)$. Thus, we have

$$
\begin{aligned}
& \dot{z}\left(t_{k}\right)=-b z\left(t_{k}\right)+\frac{1}{2} \sin 2 \phi\left(t_{k}\right) \rho^{2}\left(t_{k}\right)=0 \longrightarrow \\
& z\left(t_{k}\right)=\frac{1}{2 b} \sin 2 \phi\left(t_{k}\right) \rho^{2}\left(t_{k}\right) ; \quad k=0,1,2, \ldots
\end{aligned}
$$


As $\sin \psi=\sin (\psi+2 \pi)(\cos \psi=\cos (\psi+2 \pi))$, then it is clear that $\phi\left(t_{k+2}\right)-\phi\left(t_{k}\right)= \pm \pi, k=0,2,4, \ldots$.

The second equation of system (42) on interval $\left[t_{k}, t_{k+2}\right]$ may be also written in the integral form as

$$
\begin{aligned}
& \rho\left(t_{k+2}\right) \\
& =\rho\left(t_{k}\right) \exp \left(-\frac{\sigma+1}{2}\left(t_{k+2}-t_{k}\right)-\frac{\sigma-1}{2} \int_{t_{k}}^{t_{k+2}} \cos 2 \phi(\tau) d \tau\right. \\
& \left.+\frac{\sigma+r}{2} \int_{t_{k}}^{t_{k+2}} \sin 2 \phi(\tau) d \tau\right) \\
& \times \exp \left(-\frac{1}{2} \int_{t_{k}}^{t_{k+2}} z(\tau) \sin 2 \phi(\tau) d \tau\right) .
\end{aligned}
$$

Introduce the designations $\rho_{k}=\rho\left(t_{k}\right)$ and

$$
\begin{aligned}
& \lambda_{k}=\lambda\left(t_{k}\right) \\
&=\exp \left(-\frac{\sigma+1}{2}\left(t_{k+2}-t_{k}\right)-\frac{\sigma-1}{2} \int_{t_{k}}^{t_{k+2}} \cos 2 \phi(\tau) d \tau\right. \\
&\left.\quad+\frac{\sigma+r}{2} \int_{t_{k}}^{t_{k+2}} \sin 2 \phi(\tau) d \tau\right) .
\end{aligned}
$$

Then taking account of (46) formula (47) may be transformed as

$$
\rho_{k+2}=\lambda_{k} \rho_{k} \exp \left(-\frac{1}{4 b} \rho^{2}\left(\xi_{k}\right) \int_{t_{k}}^{t_{k+2}} \sin ^{2} 2 \phi(\tau) d \tau\right),
$$

where $t_{k}<\xi_{k}<t_{k+2}$.

Introduce the variable

$$
w_{k}=\left(\frac{1}{4 b} \int_{t_{k}}^{t_{k+2}} \sin ^{2} 2 \phi(\tau) d \tau\right)^{1 / 2} \cdot \rho\left(\xi_{k}\right)=h_{k} \cdot \rho\left(\xi_{k}\right) \text {. }
$$

By virtue of Theorem 4 for the large enough $k$ we can consider that $\lambda_{k} \approx \lambda=$ const $_{1}>0, h_{k} \approx h=$ const $_{2}>0$, and $\rho\left(\xi_{k}\right) \approx \rho\left(t_{k}\right)=\rho_{k}$.

Then from (49) it follows that

$$
\begin{array}{r}
h \rho_{k+2}=\lambda h \rho_{k} \exp \left(-h^{2} \rho_{k}^{2}\right) \longrightarrow w_{k+2}=\lambda w_{k} \exp \left(-w_{k}^{2}\right), \\
k=0,2,4, \ldots .
\end{array}
$$

As it is shown in [6] the iterated process (51) at suitable $\lambda>0$ is chaotic. Thus, this process defines a chaotic behavior of system (42) and (41).

A next obvious corollary of Theorem 6 has a large practical application.

Corollary 7. Let $\mathbb{P}$ be a vector of parameters of system (36) for which the conditions of Theorem 6 are valid. Then for any sufficiently small $\mathbb{Q}$-perturbation of the vector $\mathbb{P}$ in system (36) $(\|\mathbb{P}-\mathbb{Q}\| \approx 0)$ the perturbed system (36) with vector parameters $\mathbb{Q}$ has the same type of chaos.
Consider instead of system (30) more simple system

$$
\begin{aligned}
\dot{x}(t)= & h_{11} x(t)+h_{12} y(t)+h_{13} z(t)+a_{11} y^{2}(t) \\
& +a_{12} y(t) z(t)+a_{22} z^{2}(t), \\
\dot{y}(t)= & h_{21} x(t)+h_{22} y(t)+h_{23} z(t)+b_{12} x(t) y(t) \\
& +b_{13} x(t) z(t), \\
\dot{z}(t)= & h_{31} x(t)+h_{32} y(t)+h_{33} z(t)+c_{12} x(t) y(t) \\
& +c_{13} x(t) z(t) .
\end{aligned}
$$

Taking into account the formulas $x=\rho \cos \phi$ and $y=$ $\rho \sin \phi$, we calculate the following functions:

$$
\begin{aligned}
\Theta_{1}(y, z) & \\
= & \rho^{2} \Delta_{1}(\cos \phi, \sin \phi) \\
\equiv & \rho^{2}\left(h_{11} \cdot h(\cos \phi, \sin \phi)-f(\cos \phi, \sin \phi)\right. \\
& \cdot g(\cos \phi, \sin \phi)) \\
= & \left(h_{11} h_{22}-h_{21} h_{12}\right) y^{2}+\left(h_{11} h_{33}-h_{31} h_{13}\right) z^{2} \\
& +\left[\left(h_{11} h_{23}-h_{13} h_{21}\right)+\left(h_{11} h_{32}-h_{12} h_{31}\right)\right] y z \neq \equiv 0, \\
\Theta_{2}(y, z) & \rho^{2} \Delta_{2}(\cos \phi, \sin \phi) \\
\equiv & \rho^{2} f_{22}(\cos \phi, \sin \phi) \cdot g_{12}(\cos \phi, \sin \phi) \\
= & \left(a_{22} y^{2}+a_{23} y z+a_{33} z^{2}\right) \\
& \cdot\left[b_{12} y^{2}+\left(b_{13}+c_{12}\right) y z+c_{13} z^{2}\right] \neq \equiv .
\end{aligned}
$$

Corollary 8. Assume that for system (52) the following conditions,

(i) the forms $\Theta_{1}(y, z)$ and $\Theta_{2}(y, z)$ are nonsingular;

(ii) $h_{11}<0$;

(iii) either the quadratic form $\Theta_{1}(y, z)$ is an alternating function or $\Theta_{1}(y, z)$ is negative definite and $h_{21}^{2}+h_{31}^{2} \neq$ 0 ;

(iv) $\Theta_{2}(y, z)$ is nonpositive definite; it means that either $\Theta_{2}(y, z)=-\left(a_{22} y^{2}+a_{23} y z+a_{33} z^{2}\right)^{2}=-\left(b_{12} y^{2}+\left(b_{13}+\right.\right.$ $\left.\left.c_{12}\right) y z+c_{13} z^{2}\right)^{2}$ or one of the quadratic forms $a_{22} y^{2}+$ $a_{23} y z+a_{33} z^{2}$ or $b_{12} y^{2}+\left(b_{13}+c_{12}\right) y z+c_{13} z^{2}$ is nonpositive and other form is nonnegative, are fulfilled. Then under condition (39) (where $\left.\rho(t)=\sqrt{y^{2}(t)+z^{2}(t)}\right)$ in system (52) there is chaotic dynamics.

At research of Lorenz-like and Chen-like attractors there is a situation when one of equations of describing 
the dynamics of the corresponding system is linear (see [32, 33]). This system can be represented in the following way:

$$
\begin{gathered}
\dot{x}(t)=h_{11} x(t)+h_{12} y(t)+h_{13} z(t), \\
\dot{y}(t)=h_{21} x(t)+h_{22} y(t)+h_{23} z(t)+b_{12} x(t) y(t) \\
+b_{13} x(t) z(t), \\
\dot{z}(t)=h_{31} x(t)+h_{32} y(t)+h_{33} z(t)+c_{12} x(t) y(t) \\
+c_{13} x(t) z(t) .
\end{gathered}
$$

Introduce the matrix

$$
D=\left(\begin{array}{ll}
b_{12} & b_{13} \\
c_{12} & c_{13}
\end{array}\right) .
$$

Let $\operatorname{det} D \neq 0$. By means of approaching a linear invertible real replacement of variables $y$ and $z$ the matrix $D$ can be reduced to the normal Frobenius form as follows:

$$
D=\left(\begin{array}{cc}
0 & 1 \\
-q & -p
\end{array}\right),
$$

where $p=-b_{12}-c_{13}$ and $q=\operatorname{det} D$.

Thus, system (54) may be transformed to the following system:

$$
\begin{gathered}
\dot{x}(t)=h_{11} x(t)+h_{12} y(t)+h_{13} z(t), \\
\dot{y}(t)=h_{21} x(t)+h_{22} y(t)+h_{23} z(t)+x(t) z(t), \\
\dot{z}(t)=h_{31} x(t)+h_{32} y(t)+h_{33} z(t) \\
-q x(t) y(t)-p x(t) y(t) z(t) .
\end{gathered}
$$

(For simplicity we have left the former designations of variables $y$ and $z$ and corresponding coefficients.)

For system (57) we suppose $x=\rho \cos \phi$ and $z=\rho \sin \phi$. Then we get

$$
\begin{aligned}
\dot{y}(t)= & h_{22} y(t)+\left(h_{21} \cos \phi(t)+h_{23} \sin \phi(t)\right) \rho(t) \\
& +\cos \phi(t) \sin \phi(t) \rho^{2}(t), \\
\dot{\rho}(t)= & \left(h_{12} \cos \phi(t)+h_{32} \sin \phi(t)\right) y(t) \\
& +\left[h_{11} \cos ^{2} \phi(t)+\left(h_{31}+h_{13}\right) \cos \phi(t) \sin \phi(t)\right. \\
& \left.+h_{33} \sin ^{2} \phi(t)\right] \rho(t)-q \cos \phi(t) \sin \phi(t) y(t) \rho(t) \\
& -p \sin ^{2} \phi(t) \cos \phi(t) \rho^{2}(t), \\
\dot{\phi}(t)= & \left.-h_{12} \sin ^{2}(t)+h_{32} \cos \phi(t)\right) \frac{y(t)}{\rho(t)} \\
+ & {\left[h_{31} \cos ^{2} \phi(t)-h_{13} \sin ^{2} \phi(t)\right.} \\
& \left.+\left(h_{33}-h_{11}\right) \cos \phi(t) \sin \phi(t)\right] \\
& -q \cos ^{2} \phi(t) y(t)-p \cos ^{2} \phi(t) \sin \phi(t) \rho(t) .
\end{aligned}
$$

Note that the structure of the first two equations of system (58) is the same as that in system (37). Therefore, we can introduce the following functions:

$$
\begin{aligned}
& \Psi_{1}(x, z) \\
& =\rho^{2} \Delta_{1}(\cos \phi, \sin \phi) \\
& \equiv \rho^{2}\left(h_{22} \cdot h(\cos \phi, \sin \phi)-f(\cos \phi, \sin \phi)\right. \\
& \quad g(\cos \phi, \sin \phi)) \\
& =\left(h_{11} h_{22}-h_{21} h_{12}\right) x^{2}+\left(h_{22} h_{33}-h_{23} h_{32}\right) z^{2} \\
& +\left[h_{22} h_{31}-h_{21} h_{32}+h_{22} h_{13}-h_{23} h_{12}\right] x z \not \equiv 0, \\
& \Psi_{2}(x, z)=\rho^{2}\left(g_{22}^{2}(\cos \phi, \sin \phi)+4 \Delta_{2}(\cos \phi, \sin \phi)\right) \\
& =\left(p^{2} \sin ^{2} \phi(t)-4 q\right) x^{2} z^{2} \neq \equiv 0 .
\end{aligned}
$$

(It is clear that if $p^{2}-4 q<0$, then the form $\Psi_{2}(x, z)$ is nonpositive definite.)

Corollary 9. Assume that for system (57) the following conditions,

(i) the form $\Psi_{1}(x, z)$ is nonsingular;

(ii) $h_{22}<0$;

(iii) either the quadratic form $\Psi_{1}(x, z)$ is an alternating function or $\Psi_{1}(x, z)$ is negative definite and $h_{12}^{2}+h_{32}^{2} \neq$ 0

(iv) $p^{2}-4 q<0$,

are fulfilled. Then under condition (39), (where $\rho(t)=$ $\left.\sqrt{x^{2}(t)+z^{2}(t)}\right)$ in system (57) there is chaotic dynamics.

System (54) may be also transformed to the following system:

$$
\begin{gathered}
\dot{x}(t)=h_{11} x(t)+h_{12} y(t)+h_{13} z(t), \\
\dot{y}(t)=h_{21} x(t)+h_{22} y(t)+h_{23} z(t)-p x(t) y(t) \\
-q x(t) z(t), \\
\dot{z}(t)=h_{31} x(t)+h_{32} y(t)+h_{33} z(t)+x(t) y(t) .
\end{gathered}
$$

If for system (60) we suppose $x=\rho \cos \phi$ and $y=\rho \sin \phi$, then system (37) takes the following form:

$$
\begin{aligned}
\dot{z}(t)= & h_{33} z(t)+\left(h_{31} \cos \phi(t)+h_{32} \sin \phi(t)\right) \rho(t) \\
& +\cos \phi(t) \sin \phi(t) \rho^{2}(t), \\
\dot{\rho}(t)= & \left(h_{13} \cos \phi(t)+h_{23} \sin \phi(t)\right) z(t) \\
+ & {\left[h_{11} \cos ^{2} \phi(t)+\left(h_{21}+h_{12}\right) \cos \phi(t) \sin \phi(t)\right.} \\
& \left.+h_{22} \sin ^{2} \phi(t)\right] \rho(t)-q \cos \phi(t) \sin \phi(t) x(t) \rho(t) \\
& -p \sin ^{2} \phi(t) \cos \phi(t) \rho^{2}(t) .
\end{aligned}
$$




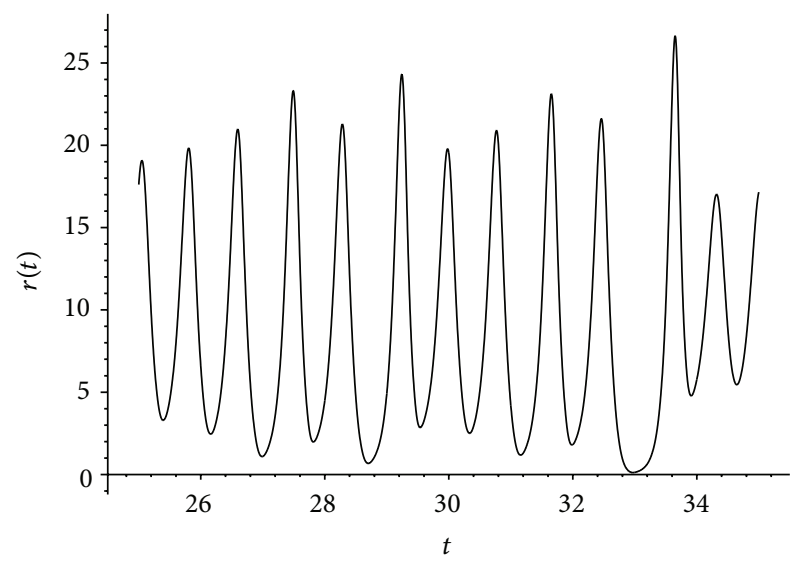

FIgURE 1: The dynamical behavior of polar radius for system (42).

Taking advantage of method of computation of functions $\Psi_{1}(x, z)$ and $\Psi_{2}(x, z)$, we can compute similar functions for system (61) as follows:

$$
\begin{aligned}
& \Omega_{1}(x, y)=\left(h_{11} h_{33}-h_{31} h_{13}\right) x^{2}+\left(h_{22} h_{33}-h_{23} h_{32}\right) y^{2} \\
&+\left[h_{12} h_{33}-h_{13} h_{32}+h_{21} h_{33}-h_{23} h_{31}\right] x y \\
& \Omega_{2}(x, y)=\left(p^{2} \sin ^{2} \phi(t)-4 q\right) x^{2} y^{2} .
\end{aligned}
$$

If we change $z \rightarrow y, h_{22} \rightarrow h_{33}, h_{12} \rightarrow h_{21}, h_{32} \rightarrow h_{31}$, $\Psi_{1}(x, z) \rightarrow \Omega_{1}(x, y)$, and $\Psi_{2}(x, z) \rightarrow \Omega_{2}(x, y)$, then for system (60) the conditions of Corollary 9 remain valid.

\section{Examples}

(1) For Lorenz's system (41) represented as (57) we have $h_{22}=$ $-1<0, p=0$, and $q=1$ and the form $\Psi_{1}(x, z)=(1-$ r) $\sigma x^{2}+b z^{2}$ is an alternating function. Thus, the conditions (i) -(iv) of Corollary 9 are fulfilled. (For system (60) we obtain $h_{33}=-b<0, p=0$, and $q=1$ and the form $\Omega_{1}(x, y)=$ $b \sigma x^{2}+b y^{2}-b(\sigma+r) x y$ is an alternating function.) On Figure 1 the dynamics of polar radius for system (42) is shown.

It is easy to check that conditions (i)-(ii) of Theorem 6 and also condition (39) are valid. (Indeed, for system (42) on Figure 1 at $t_{m}^{*}=33.01$, we have $\rho\left(t_{m}^{*}\right) \approx 0$.)

(2) Consider the system [12]

$$
\begin{gathered}
\dot{x}(t)=-\frac{a b}{a+b} x(t)-y(t) z(t)+c, \\
\dot{y}(t)=a y(t)+x(t) z(t), \\
\dot{z}(t)=b z(t)+x(t) y(t),
\end{gathered}
$$

where $a, b$, and $c$ are real numbers.

Let $A(d, e, f)$ be an equilibrium point of system (63). (The real numbers $x=d, y=e$, and $z=f$ are determined from the system of the following equations: $-a b x /(a+b)-y z+c=$ $0, a y+x z=0, b z+x y=0$.)
Introduce in system (63) new coordinates under the formulas $x \rightarrow x-d, y \rightarrow 2 y-e$, and $z \rightarrow z-f$. Then the system (63) can be transformed to the following system:

$$
\begin{gathered}
\dot{x}(t)=-\frac{a b}{a+b} x(t)+2 f y(t)+e z(t)-2 y(t) z(t), \\
\dot{y}(t)=-\frac{1}{2} f x(t)+a y(t)-\frac{1}{2} d z(t)+\frac{1}{2} x(t) z(t), \\
\dot{z}(t)=-e x(t)-2 d y(t)+b z(t)+2 x(t) y(t)
\end{gathered}
$$

with origin of coordinates in the point $A$. (For simplicity we saved former designations of system (63) in system (64).)

Now we introduce in system (64) polar coordinates under the formulas $x=\rho \cos \phi$ and $y=\rho \sin \phi$. Then we have

$$
\begin{aligned}
\dot{z}(t)= & b z-(e \cos \phi+2 d \sin \phi) \rho+(\sin 2 \phi) \rho^{2}, \\
\dot{\rho}(t)= & \left(e \cos \phi-\frac{1}{2} d \sin \phi\right) z \\
& +\left(a \sin ^{2} \phi+\frac{3}{4} f \sin 2 \phi-\frac{a b}{a+b} \cos ^{2} \phi\right) \rho \\
\dot{\phi}(t)= & \frac{a b}{2(a+b)} \sin 2 \phi-2 f \sin ^{2} \phi-\frac{1}{2} f \cos ^{2} \phi+\frac{1}{2} a \sin 2 \phi \\
+ & \left.(2 \sin 2 \phi) z \rho, \frac{1}{2} \cos ^{2} \phi\right) z \\
- & \frac{(e \sin \phi+(1 / 2) d \cos \phi) z}{\rho} .
\end{aligned}
$$

System (65) is a special case of system (36). Besides, the subsystem consisting of the first two equations of system (65) is a special case of system (37).

Let $a=-12, b=-6$, and $c=-15$. Then we have $d=$ $3.75, e=0.0$, and $f=0.0$. It is easy to check that conditions (i)-(ii) of Theorem 6 are valid. It is yet necessary to check up condition (39).

Consider the dynamic change of polar radius for system (65) (see Figure 2). Then we have that dynamical behavior in Figure 2.

It is clear that for $t_{m_{1}}^{*}=6.42, t_{m_{2}}^{*}=9.31$, and $t_{m_{3}}^{*}=13.19$ condition (39) $\left(\rho\left(t_{m_{i}}^{*}\right) \approx 0\right)$ is fulfilled. On Figure 3 the chaotic attractor of systems (64) is shown.

(3) Consider the following system:

$$
\begin{gathered}
\dot{x}(t)=-2 x(t)+b y^{2}(t)+4 z^{2}(t), \\
\dot{y}(t)=\mu x(t)+c y(t)+d z(t)-e x(t) y(t), \\
\dot{z}(t)=\mu x(t)-d y(t)+c z(t)-e x(t) z(t) .
\end{gathered}
$$




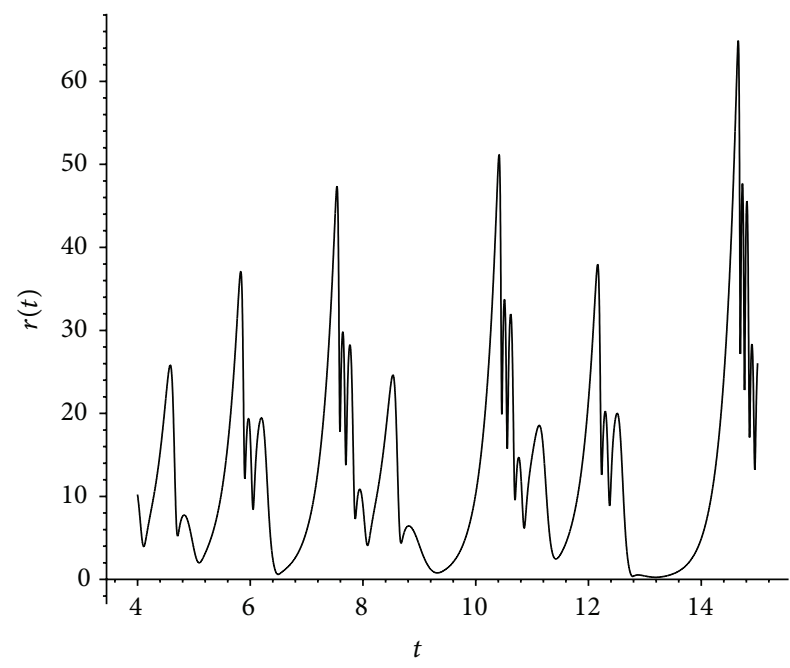

FIGURE 2: The dynamical behavior of polar radius for system (65).

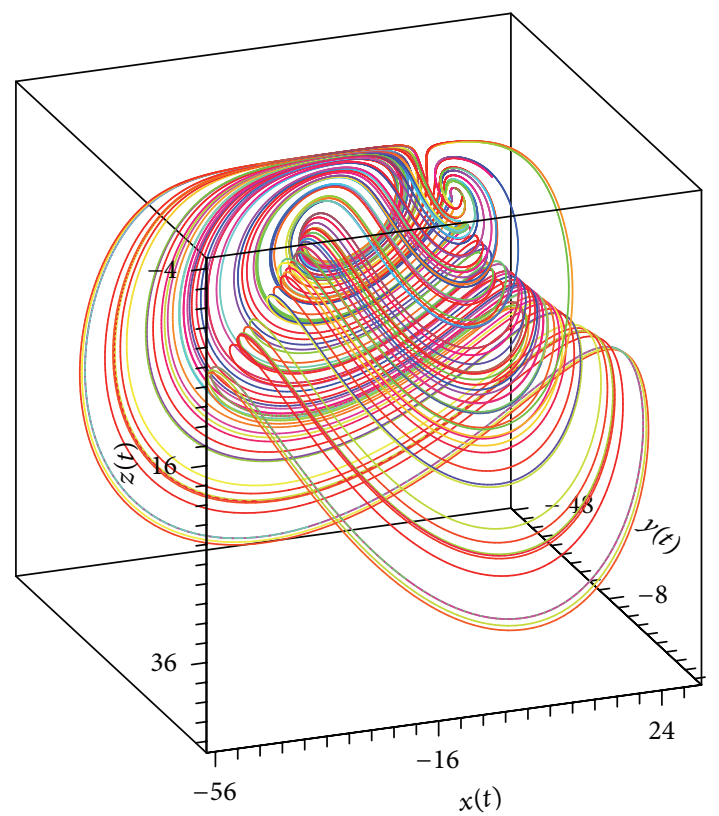

FIgURE 3: The chaotic attractor of system (64).

In the polar coordinates system (66) takes the following form (see (16)):

$$
\begin{gathered}
\dot{x}(t)=-2 x+\left(b \sin ^{2} \phi+4 \cos ^{2} \phi\right) \cdot \rho^{2}, \\
\dot{\rho}(t)=\mu \cdot(\cos \phi+\sin \phi) \cdot x+c \rho-\operatorname{ex} \rho, \\
\dot{\phi}(t)=-d-\mu \cdot(\cos \phi-\sin \phi) \cdot \frac{x}{\rho} .
\end{gathered}
$$

Let initial data be $x_{0}=1, \rho_{0}=1$, and $\phi_{0}=0$. It is easy to check that the conditions of Theorems 5 and 6 at $\mu \in[0,2.07)$ and $b=7, c=4, d=7$, and $e=10$ are fulfilled. Thus, $\mu^{*}=2.07$.

On Figures 4-10 different characteristics of system (66) are shown.

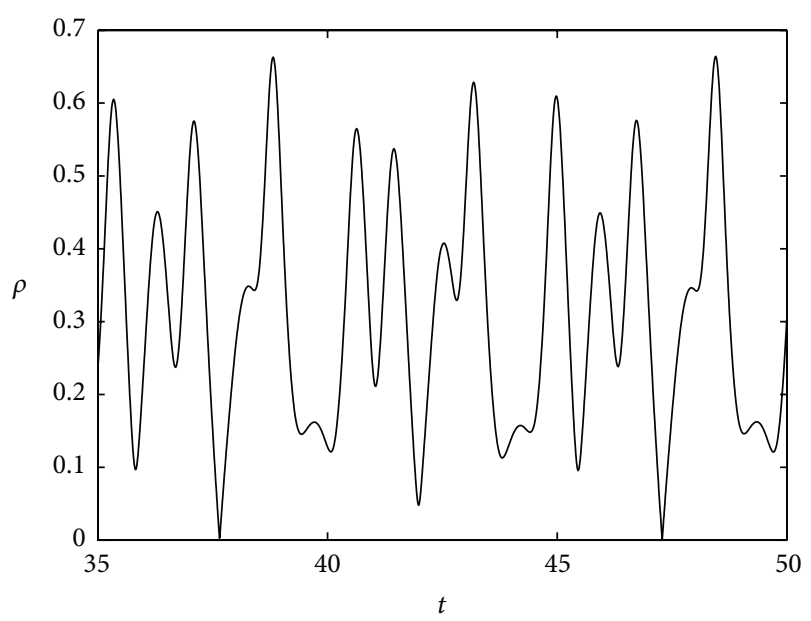

FIgURE 4: The dependence of radius $\rho$ on time $t$ for system (67) at $\mu=2, b=7, c=4, d=7$, and $e=10$. (It is clear that for $t_{m_{1}}^{*}=37.60$, $t_{m_{2}}^{*}=47.41$ condition $(39)\left(\rho\left(t_{m_{i}}^{*}\right) \approx 0\right)$ is fulfilled.)

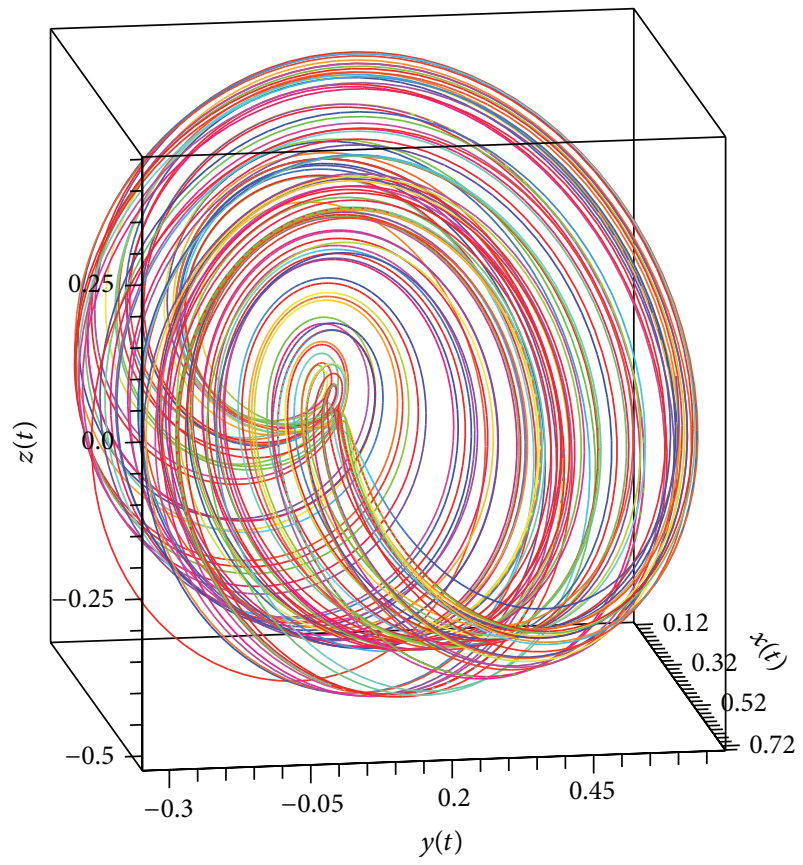

FIGURE 5: The chaotic attractor of system (66) at $\mu=2, b=7, c=4$, $d=7$, and $e=10$.

From Figures 6-10 it follows that in domain of chaos one of the Lyapunov exponents is positive, another is zero, and third is negative. If two exponents are negative and one exponent is zero, then in system (66) there is a limit cycle.

(4) Consider the Liu system [13]

$$
\begin{aligned}
& \dot{x}(t)=a x(t)+d_{1} y(t) z(t), \\
& \dot{y}(t)=b y(t)+d_{2} x(t) z(t), \\
& \dot{z}(t)=c z(t)+d_{3} x(t) y(t) .
\end{aligned}
$$



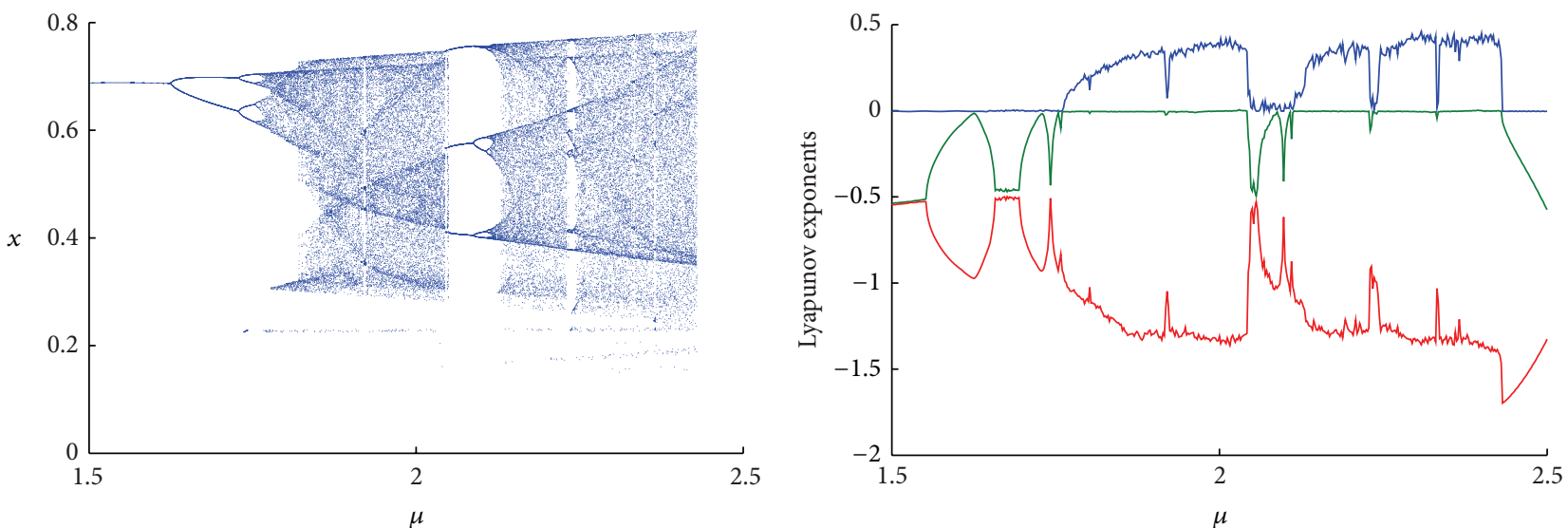

FIGURE 6: The bifurcation diagram and Lyapunov exponents of system (66) at $b=7, c=4, d=7$, and $e=10$.
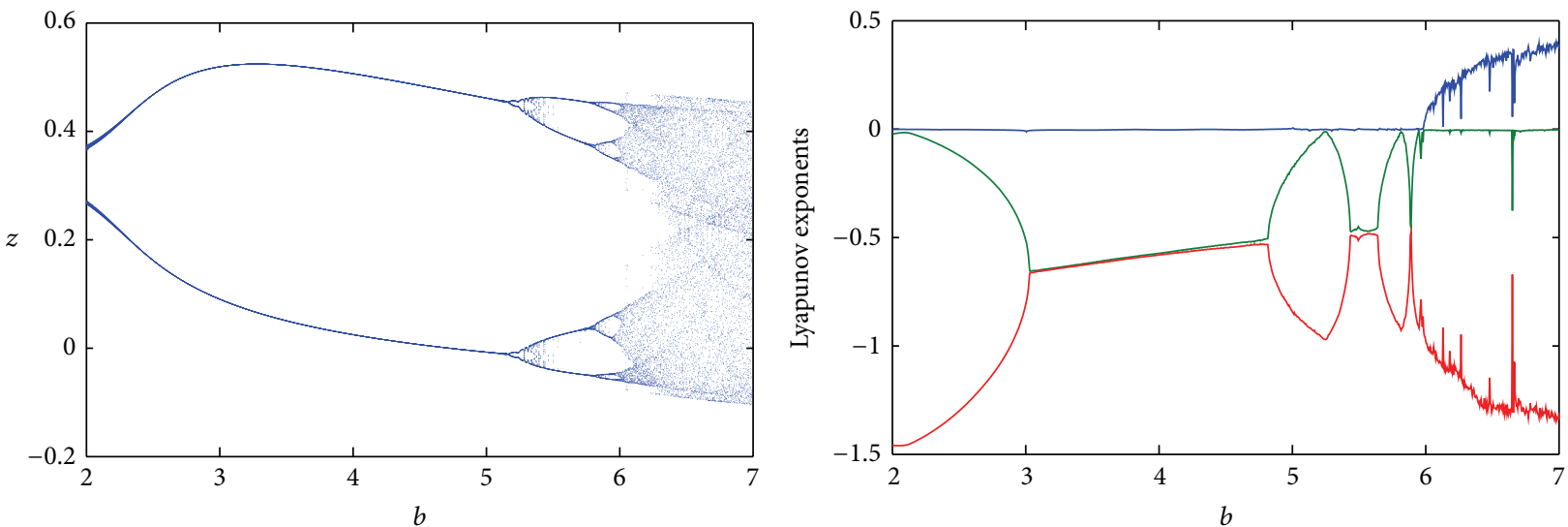

Figure 7: The bifurcation diagram and Lyapunov exponents of system (66) at $\mu=2, c=4, d=7$, and $e=10$.
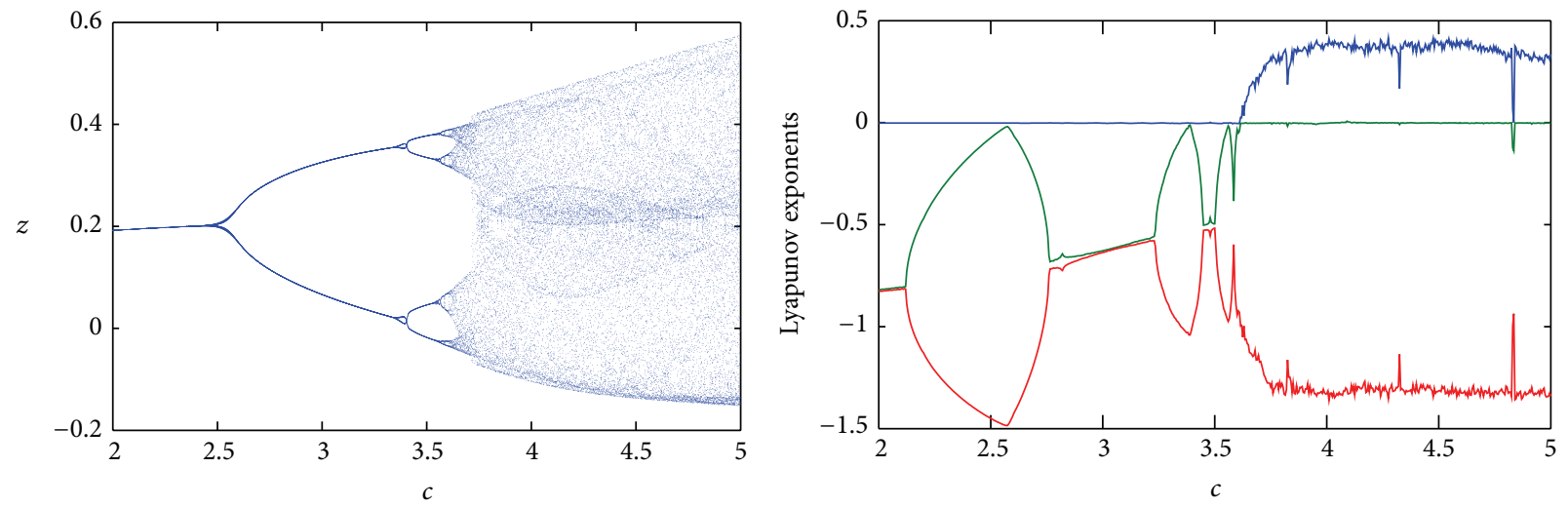

Figure 8: The bifurcation diagram and Lyapunov exponents of system (66) at $\mu=2, b=7, d=7$, and $e=10$. form:

In the polar coordinates system (68) takes the following

$$
\begin{gathered}
\dot{x}(t)=a x+d_{1} \cdot \rho^{2} \sin \phi \cdot \cos \phi, \\
\dot{\rho}(t)=\left(c \sin ^{2} \phi+b \cos ^{2} \phi\right) \cdot \rho+\left(d_{2}+d_{3}\right) \cdot x \rho \sin \phi \cdot \cos \phi, \\
\dot{\phi}(t)=(c-b) \cdot \cos \phi \cdot \sin \phi+\left(-d_{2} \sin ^{2} \phi+d_{3} \cos ^{2} \phi\right) \cdot x .
\end{gathered}
$$

It is easy to check that the conditions of Theorem 6 at $a=-4, b=-1.3, c=1.2, d_{1}=4, d_{2}=1$, and $d_{3}=-2$ are fulfilled. The behavior of the attractor of system (68) is shown on Figures 11 and 12.

(5) Consider the Chen system [13]

$$
\begin{gathered}
\dot{x}(t)=a(y(t)-x(t)), \\
\dot{y}(t)=(c-a) x(t)+c y(t)-x(t) z(t), \\
\dot{z}(t)=-b z(t)+x(t) y(t) .
\end{gathered}
$$



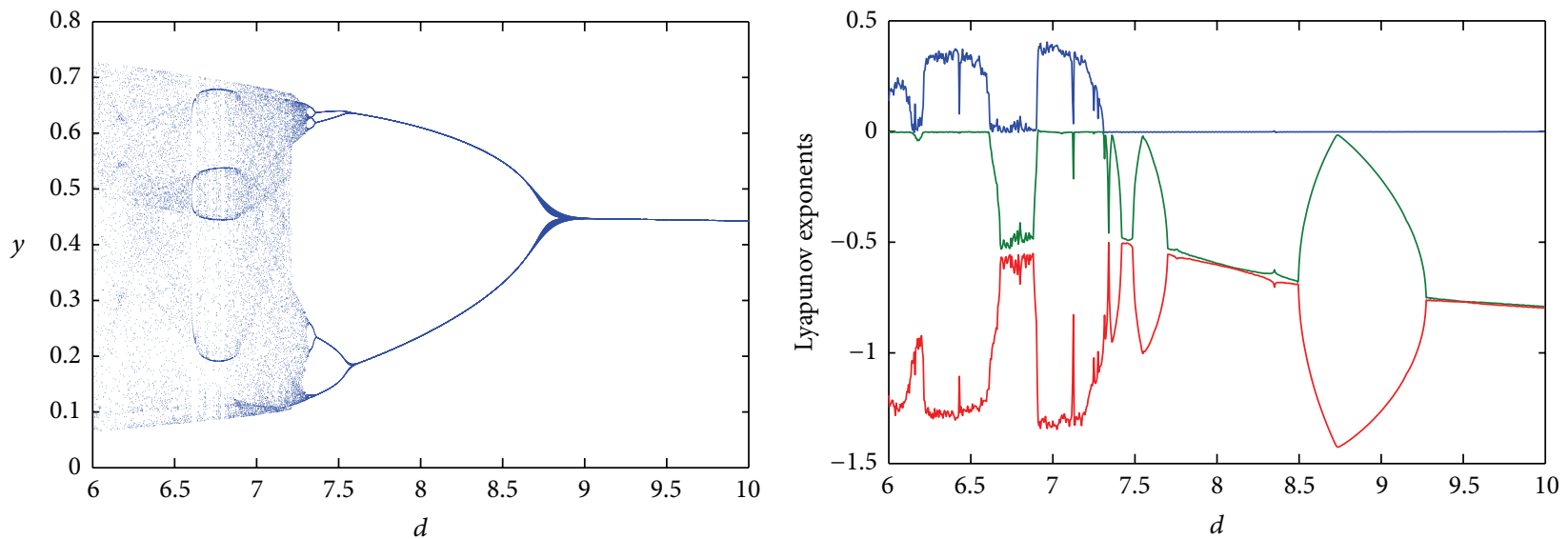

Figure 9: The bifurcation diagram and Lyapunov exponents of system (66) at $\mu=2, b=7, c=4$, and $e=10$.
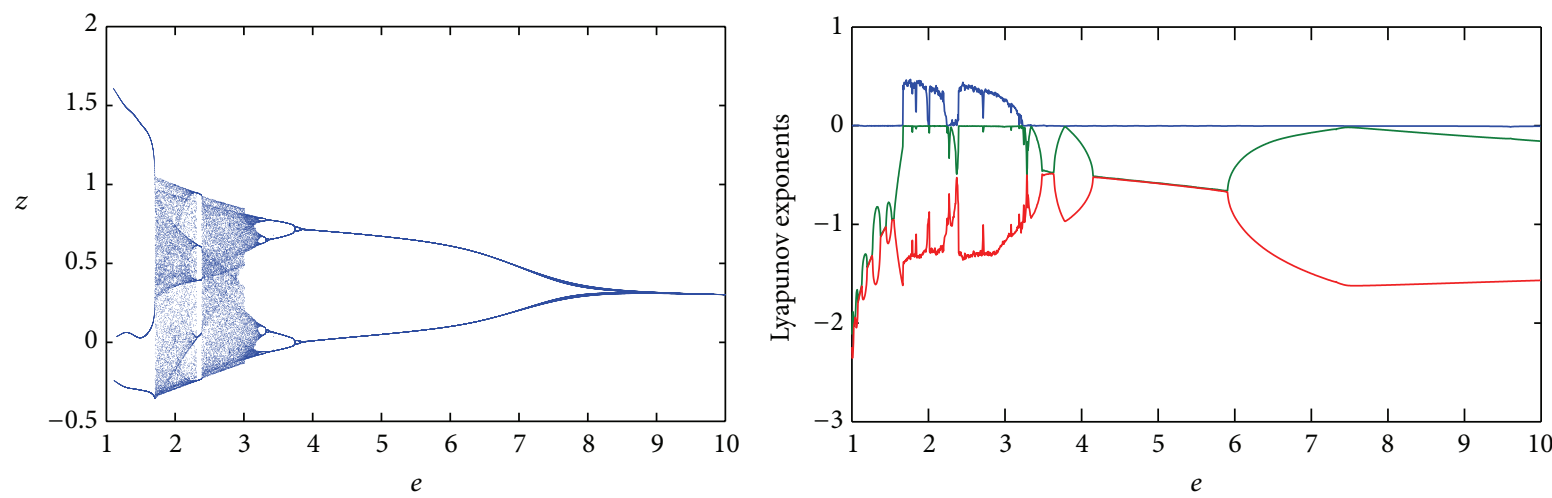

FIGURE 10: The bifurcation diagram and Lyapunov exponents of system (66) at $\mu=2, b=7, c=7$, and $d=10$.

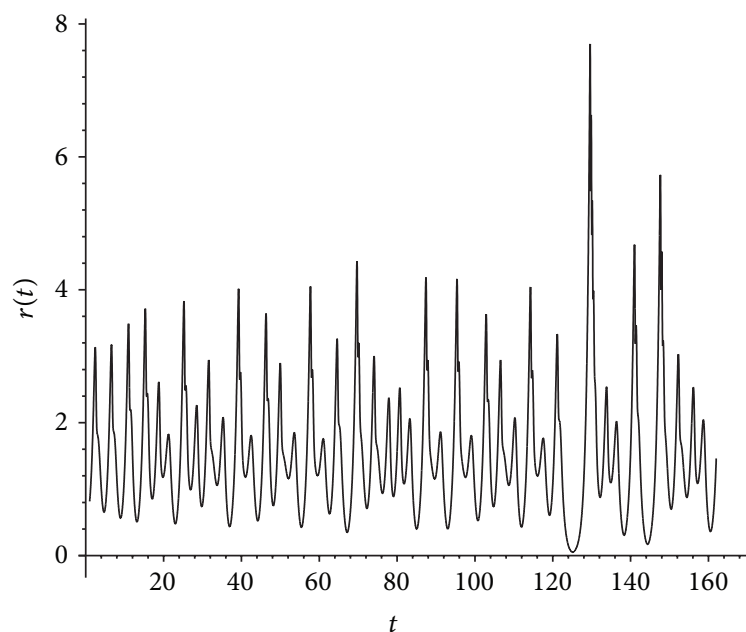

FIgURE 11: The dependence of radius $\rho$ on time $t$ for system (69). Here for $t_{m}^{*}=125$ condition (39) $\left(\rho\left(t_{m}^{*}\right) \approx 0\right)$ is fulfilled. form:

In the polar coordinates system (70) takes the following

$$
\begin{gathered}
\dot{z}(t)=-b z+\rho^{2} \sin \phi \cdot \cos \phi, \\
\dot{\rho}(t)=\left(c \sin ^{2} \phi+c \sin \phi \cdot \cos \phi-a \cos ^{2} \phi\right) \\
\cdot \rho-z \cdot \rho \sin \phi \cdot \cos \phi,
\end{gathered}
$$

$$
\begin{aligned}
\dot{\phi}(t)= & -a+(a+c) \cdot \cos \phi \cdot \sin \phi+c \cdot \cos ^{2} \phi \\
& -z \cdot \cos ^{2} \phi .
\end{aligned}
$$

The conditions of Theorem 6 at $a=35, b=1$, and $c=28$ are fulfilled. The behavior of radius of system (70) is shown on Figure 13.

\section{Conclusion}

In [31] (Theorem 1) the necessary and sufficient conditions of boundedness of solutions for system (37) with constant coefficients were found. Then from these conditions in [31] (Lemma 10) the existence condition of the loop of separatrix (homoclinic orbit) was got. It is easy to show that the presence of homoclinic orbit in system (37) is a necessary condition for the existence of chaotic dynamics in system (30).

A search of the existence sufficient conditions of homoclinic orbits for system (30) is a difficult problem. Therefore another idea was realized. For the quadratic 3D system (16) the discrete $1 \mathrm{D}$ mapping was built; its state of chaos is then proved. On system (30) this approach was extended. As a result in Theorem 6 the existence conditions of chaos unknown earlier were got. 


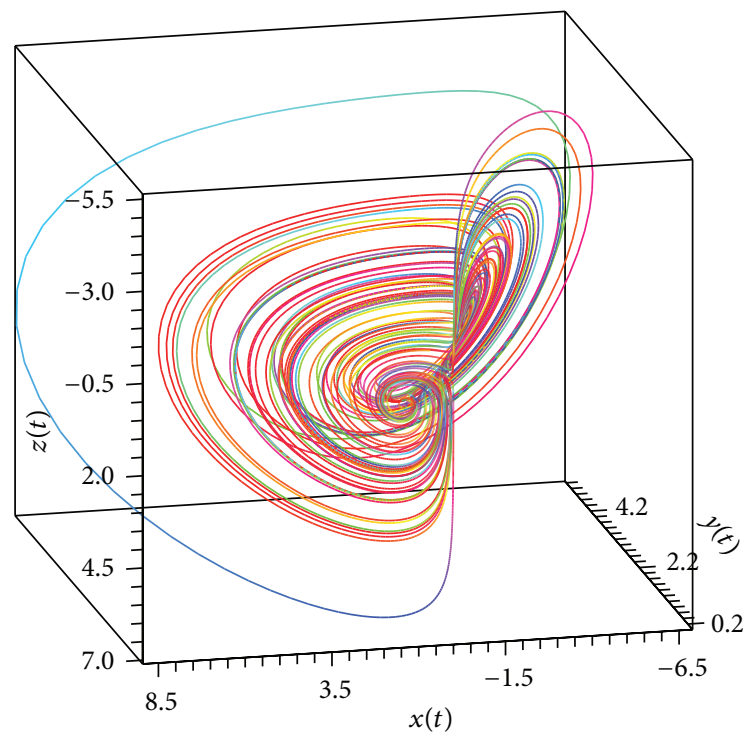

FIGURE 12: The chaotic attractor of system (68).

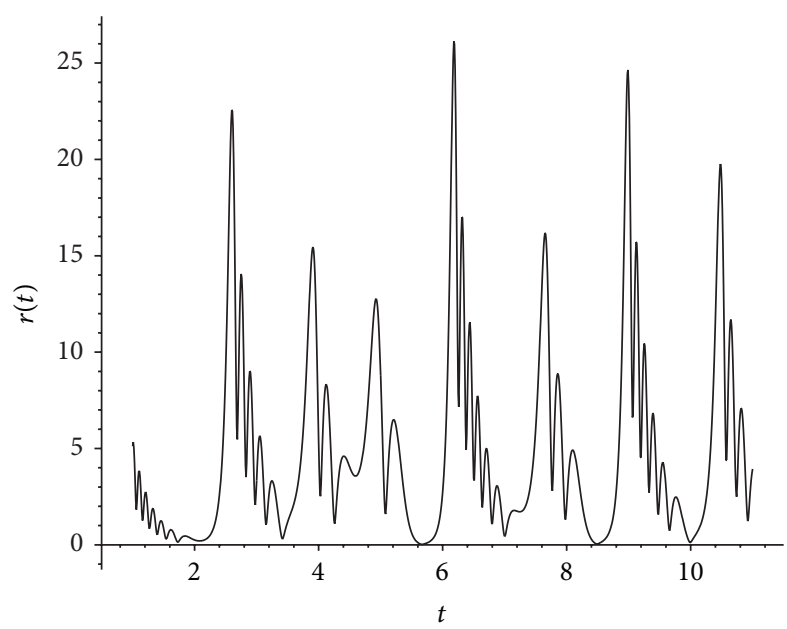

Figure 13: The dependence of radius $\rho$ on time $t$ for system (70). (It is clear that for $t_{m_{1}}^{*}=5.61, t_{m_{2}}^{*}=8.42$, and $t_{m_{3}}^{*}=10.01$ condition (39) $\left(\rho\left(t_{m_{i}}^{*}\right) \approx 0\right)$ is valid.)

\section{Conflict of Interests}

The author declares that there is no conflict of interests regarding the publication of this paper.

\section{Acknowledgment}

The author is grateful to the anonymous referee for the comments which led to an improvement of the presentation.

\section{References}

[1] F. Balibrea, T. Caraballo, P. E. Kloeden, and J. Valero, "Recent developments in dynamical systems: three perspectives," International Journal of Bifurcation and Chaos in Applied Sciences and Engineering, vol. 20, no. 9, pp. 2591-2636, 2010.
[2] U. Feudel, “Complex dynamics in multistable systems," International Journal of Bifurcation and Chaos in Applied Sciences and Engineering, vol. 18, no. 6, pp. 1607-1626, 2008.

[3] A. C. Luo and Y. Guo, "Parameter characteristics for stable and unstable solutions in nonlinear discrete dynamical systems," International Journal of Bifurcation and Chaos in Applied Sciences and Engineering, vol. 20, no. 10, pp. 3173-3191, 2010.

[4] V. Y. Belozyorov, "New types of 3-D systems of quadratic differential equations with chaotic dynamics based on Ricker discrete population model," Applied Mathematics and Computation, vol. 218, no. 8, pp. 4546-4566, 2011.

[5] V. Y. Belozyorov, "Implicit one-dimensional discrete maps and their connection with existence problem of chaotic dynamics in 3-D systems of differential equations," Applied Mathematics and Computation, vol. 218, no. 17, pp. 8869-8886, 2012.

[6] V. Y. Belozyorov and S. V. Chernyshenko, "Generating chaos in 3D systems of quadratic differential equations with $1 \mathrm{D}$ Exponential maps," International Journal of Bifurcation and Chaos in Applied Sciences and Engineering, vol. 23, no. 6, Article ID 1350105, 16 pages, 2013.

[7] V. Y. Belozyorov, "General method of construction of implicit discrete maps generating chaos in 3D quadratic systems of differential equations," International Journal of Bifurcation and Chaos in Applied Sciences and Engineering, vol. 24, no. 2, Article ID 1450025, 23 pages, 2014.

[8] L. Gardini, I. Sushko, V. Avrutin, and M. Schanz, "Critical homoclinic orbits lead to snap-back repellers," Chaos, Solitons \& Fractals, vol. 44, no. 6, pp. 433-449, 2011.

[9] R. C. Robinson, An Introduction to Dynamical Systems: Continuous and Discrete, Prentice Hall, New York, NY, USA, 2004.

[10] X. Shen and Z. Jia, "On the existence structure of onedimensional discrete chaotic systems," Journal of Mathematics Research, vol. 3, no. 3, pp. 22-27, 2011.

[11] X. Zhang, Y. Shi, and G. Chen, "Constructing chaotic polynomial maps," International Journal of Bifurcation and Chaos in Applied Sciences and Engineering, vol. 19, no. 2, pp. 531-543, 2009.

[12] J. Lu and G. Chen, "Generating multiscroll chaotic attractors: theories, methods and applications," International Journal of Bifurcation and Chaos in Applied Sciences and Engineering, vol. 16, no. 4, pp. 775-858, 2006.

[13] T. Zhou and G. Chen, "Classification of chaos in 3-D autonomous quadratic systems. I. Basic framework and methods," International Journal of Bifurcation and Chaos in Applied Sciences and Engineering, vol. 16, no. 9, pp. 2459-2479, 2006.

[14] V. Y. Belozyorov, "On existence of homoclinic orbits for some types of autonomous quadratic systems of differential equations," Applied Mathematics and Computation, vol. 217, no. 9, pp. 4582-4595, 2011.

[15] M. M. El-Dessoky, M. T. Yassen, E. Saleh, and E. S. Aly, "Existence of heteroclinic and homoclinic orbits in two different chaotic dynamical systems," Applied Mathematics and Computation, vol. 218, no. 24, pp. 11859-11870, 2012.

[16] J. Bao and Q. Yang, "A new method to find homoclinic and heteroclinic orbits," Applied Mathematics and Computation, vol. 217, no. 14, pp. 6526-6540, 2011.

[17] G. A. Leonov, "Shilnikov chaos in Lorenz-like systems," International Journal of Bifurcation and Chaos in Applied Sciences and Engineering, vol. 23, no. 3, Article ID 1350058, 10 pages, 2013.

[18] D. Shang and M. Han, "The existence of homoclinic orbits to saddle-focus," Applied Mathematics and Computation, vol. 163, no. 2, pp. 621-631, 2005. 
[19] X. Wang, "Shil'nikov chaos and Hopf bifurcation analysis of Rucklidge system," Chaos, Solitons \& Fractals, vol. 42, no. 4, pp. 2208-2217, 2009.

[20] Q. Yang, Z. Wei, and G. Chen, "An unusual 3D autonomous quadratic chaotic system with two stable node-foci," International Journal of Bifurcation and Chaos in Applied Sciences and Engineering, vol. 20, no. 4, pp. 1061-1083, 2010.

[21] Z. Zheng and G. Chen, "Existence of heteroclinic orbits of the Shil'nikov type in a 3D quadratic autonomous chaotic system," Journal of Mathematical Analysis and Applications, vol. 315, no. 1, pp. 106-119, 2006.

[22] Z. Li, G. Chen, and W. A. Halang, "Homoclinic and heteroclinic orbits in a modified Lorenz system," Information Sciences, vol. 165, no. 3-4, pp. 235-245, 2004.

[23] T. Zhou, G. Chen, and Q. Yang, "Constructing a new chaotic system based on the Shilnikov criterion," Chaos, Solitons and Fractals, vol. 19, no. 4, pp. 985-993, 2004.

[24] L. F. Mello, M. Messias, and D. C. Braga, "Bifurcation analysis of a new Lorenz-like chaotic system," Chaos, Solitons \& Fractals, vol. 37, no. 4, pp. 1244-1255, 2008.

[25] Z. Chen, Y. Yang, and Z. Yuan, "A single three-wing or four-wing chaotic attractor generated from a three-dimensional smooth quadratic autonomous system," Chaos, Solitons \& Fractals, vol. 38, no. 4, pp. 1187-1196, 2008.

[26] G. Qi, G. Chen, M. A. van Wyk, B. J. van Wyk, and Y. Zhang, "A four-wing chaotic attractor generated from a new 3-D quadratic autonomous system," Chaos, Solitons and Fractals, vol. 38, no. 3, pp. 705-721, 2008.

[27] B. Yu and G. Hu, "Constructing multiwing hyperchaotic attractors," International Journal of Bifurcation and Chaos, vol. 20, no. 3, pp. 727-734, 2010.

[28] S. Vahedi and M. S. Noorani, "Analysis of a new quadratic 3D chaotic attractor," Abstract and Applied Analysis, vol. 2013, Article ID 540769, 7 pages, 2013.

[29] Z. Wang, Z. Wei, X. Xi, and Y. Li, "Dynamics of a 3D autonomous quadratic system with an invariant algebraic surface," Nonlinear Dynamics, vol. 77, no. 4, pp. 1503-1518, 2014.

[30] H. K. Khalil, Nonlinear Systems, Prentice Hall, Upper Saddle River, NJ, USA, 2nd edition, 1996.

[31] R. J. Dickson and L. M. Perko, "Bounded quadratic systems in the plane," Journal of Differential Equations, vol. 7, pp. 251-273, 1970.

[32] Q. Yang and G. Chen, "A chaotic system with one saddle and two stable node-foci," International Journal of Bifurcation and Chaos in Applied Sciences and Engineering, vol. 18, no. 5, pp. 1393-1414, 2008.

[33] X. Wang and G. Chen, "A gallery of Lorenz-like and Chenlike attractors," International Journal of Bifurcation and Chaos in Applied Sciences and Engineering, vol. 23, no. 4, Article ID 1330011, 20 pages, 2013. 


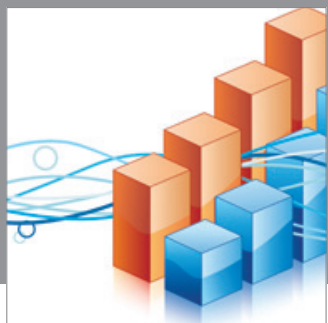

Advances in

Operations Research

mansans

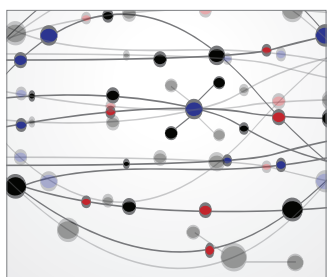

The Scientific World Journal
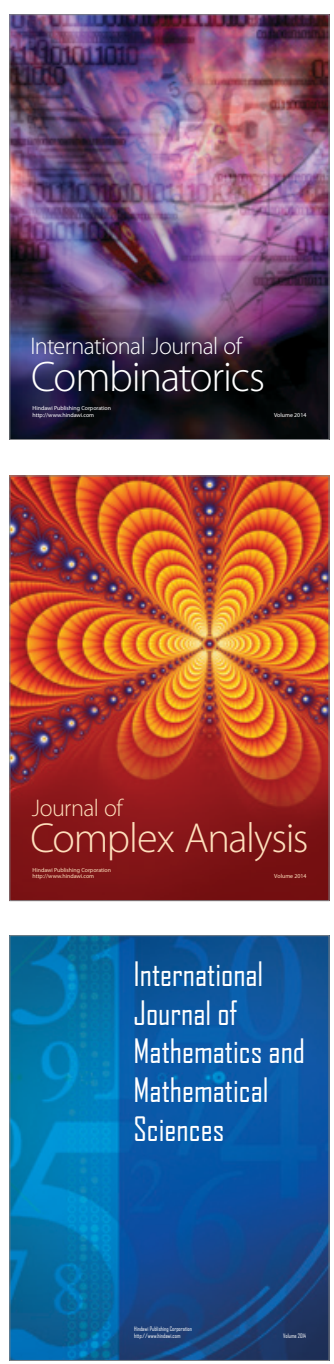
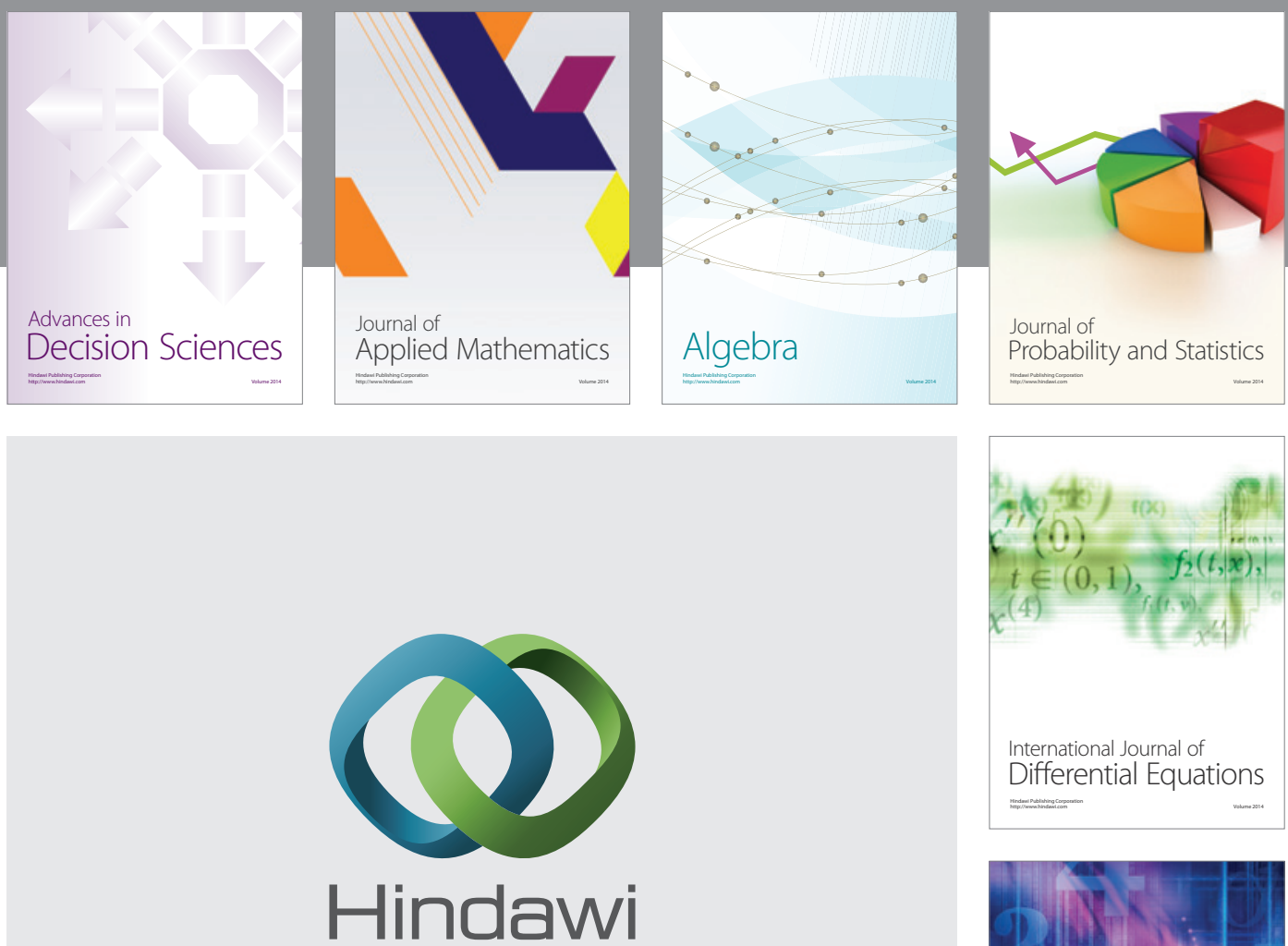

Submit your manuscripts at http://www.hindawi.com
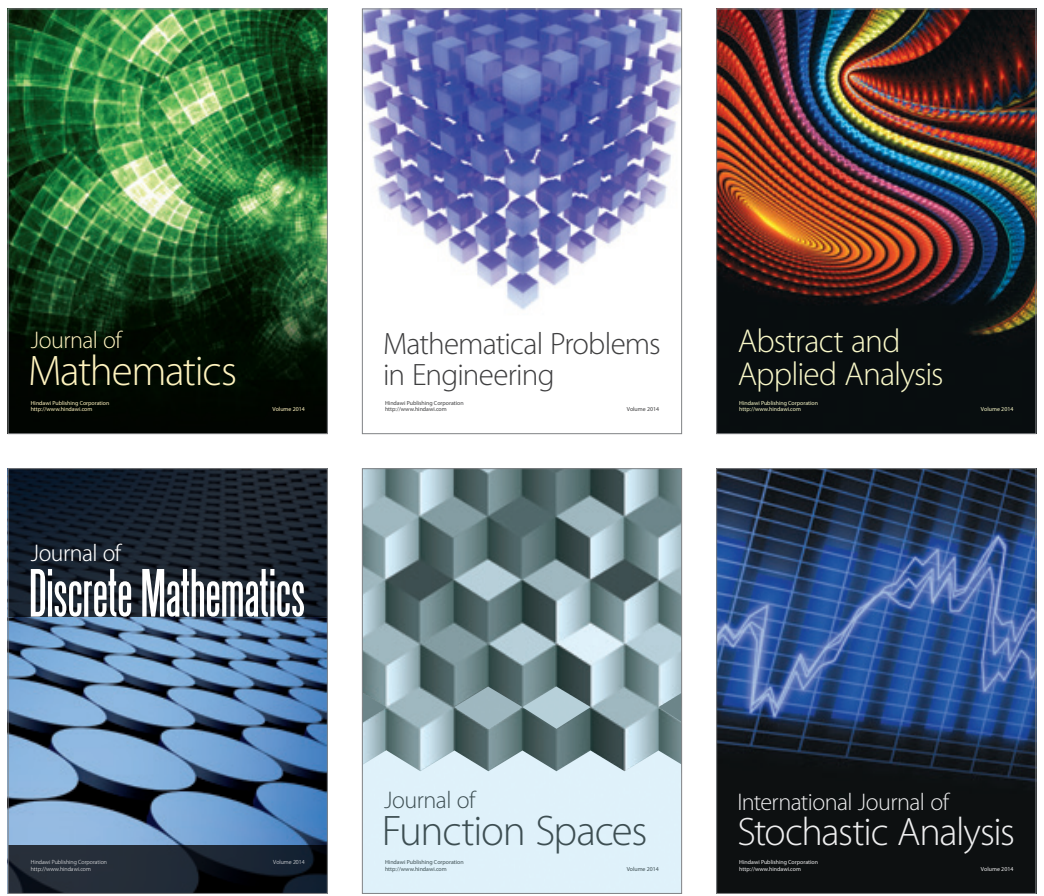

Journal of

Function Spaces

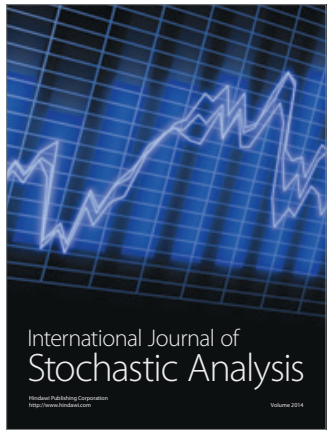

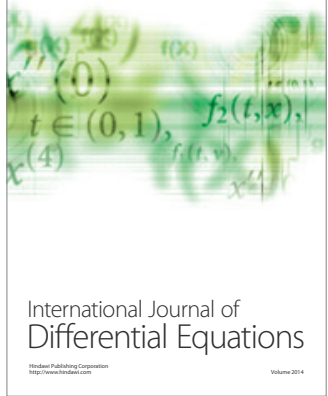
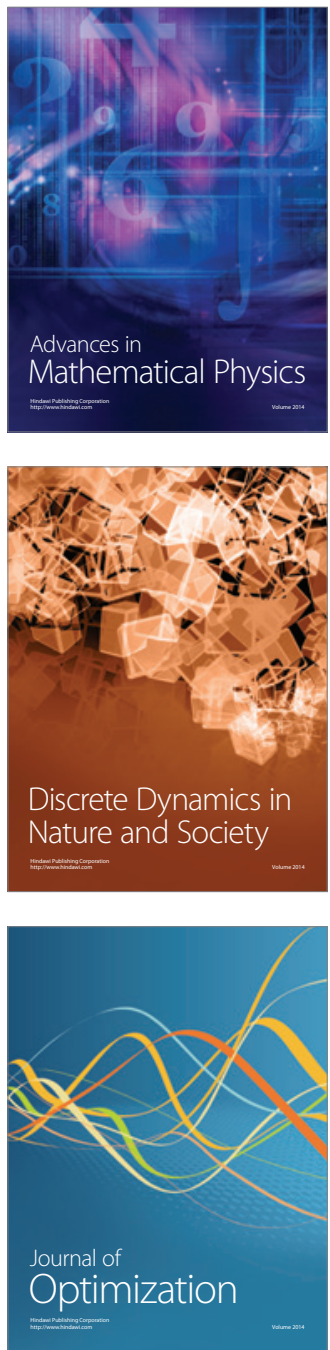